hep-th/0412185

CPTH RR 065.1104

LPT-ORSAY 04-123

UMN-TH-2333/04

December 2004

\title{
A Calculable Toy Model of the Landscape
}

\author{
Keith R. Dienes ${ }^{1 *}$, Emilian Dudas ${ }^{2,3 \dagger}$, Tony Gherghetta ${ }^{4 \ddagger}$ \\ ${ }^{1}$ Department of Physics, University of Arizona, Tucson, AZ 85721 USA \\ ${ }^{2}$ Centre de Physique Théorique, Ecole Polytechnique, \\ F-91128, Palaiseau Cedex, France \\ ${ }^{3}$ LPT, Bât. 210, Univ. Paris-Sud, F-91405, Orsay Cedex, France \\ 4 School of Physics and Astronomy, University of Minnesota, \\ Minneapolis, MN 55455 USA
}

\begin{abstract}
Motivated by recent discussions of the string-theory landscape, we propose field-theoretic realizations of models with large numbers of vacua. These models contain multiple $U(1)$ gauge groups, and can be interpreted as deconstructed versions of higher-dimensional gauge theory models with fluxes in the compact space. We find that the vacuum structure of these models is very rich, defined by parameter-space regions with different classes of stable vacua separated by boundaries. This allows us to explicitly calculate physical quantities such as the supersymmetry-breaking scale, the presence or absence of $R$-symmetries, and probabilities of stable versus unstable vacua. Furthermore, we find that this landscape picture evolves with energy, allowing vacua to undergo phase transitions as they cross the boundaries between different regions in the landscape. We also demonstrate that supergravity effects are crucial in order to stabilize most of these vacua, and in order to allow the possibility of cancelling the cosmological constant.
\end{abstract}

\footnotetext{
${ }^{*}$ E-mail address: dienes@physics.arizona.edu

${ }^{\dagger}$ E-mail address: emilian.dudas@cpht.polytechnique.fr

$\ddagger$ E-mail address: tgher@physics.umn.edu
} 


\section{Introduction}

Recent developments in the study of string-theory compactifications suggest that there exist huge numbers of string vacua, with different cosmological constants and different low-energy phenomenological properties [1]. The resulting picture, dubbed the "landscape" [2], has stimulated a statistical analysis of the number of string vacua [3], the supersymmetry-breaking scale, and other phenomenological features [4]. Anthropic principles have even been advanced to resolve difficult issues such as the cosmological constant problem [5].

One of the problems facing this landscape picture of string theory is that of calculating physical parameters. This is, to a large extent, due to the limited technology for performing string calculations in the relevant flux vacua [6]. It is therefore useful to present field-theoretic counterparts of such constructions, i.e., field-theoretic models which naturally give rise to very large numbers of vacua, and to be able to quantitatively determine statistical distributions of relevant physical quantities such as the cosmological constant, the scale of supersymmetry breaking, the Higgs mass, gauge and Yukawa couplings, and the like.

The purpose of this paper is to present such field-theoretic examples based on multiple Abelian gauge groups and multiple charged scalar fields. As we shall see, such models naturally lead to large numbers of vacua, and allow us to quantitatively address many of the pressing questions that such pictures raise.

In Sect. 2, as a brief warm-up, we discuss the standard Fayet-Iliopoulos model, now reconsidered from our perspective. In Sect. 3, we then describe, in some detail, the construction of a relatively simple toy model which nevertheless gives rise to many of the landscape features that we wish to study. Specifically, we consider a supersymmetric model with two $U(1)$ 's with Fayet-Iliopoulos terms, and three charged chiral superfields. We show that this model contains seven distinct classes of vacua which populate the parameter space in different pie-slice regions, giving rise to vastly different physical properties such as supersymmetry-breaking scale and numbers of unbroken gauge and global symmetries. We also show that renormalization-group evolution can bring the theory from one vacuum to another, while the boundaries between different regions correspond to parameter-space surfaces on which a single scalar particle becomes massless.

In Sect. 4, we consider a generalization of this toy model to more complicated supersymmetric models with large numbers of $U(1)$ gauge groups. Such models easily give rise to huge numbers of distinct classes of vacua. The simplest example is provided by $n$ copies of the Fayet-Iliopoulos model, thereby giving rise to $3^{n}$ distinct classes of vacua. We concentrate most of our efforts, however, on a four-dimensional model which we believe is the "deconstruction" [7] of a six-dimensional model with internal magnetic fluxes. Our interest in this respect is to provide a purely fourdimensional description of these higher-dimensional flux models which are among the simplest models that generate chirality in four dimensions. This flux-compactification 
framework is also probably the simplest and most successful framework for the construction of semi-realistic string models with intersecting branes [8]. We also comment on the role of mixed anomalies and their cancellation in the vacua we consider, and show that almost all of these vacua are unstable, in analogy with the Nielsen-Olesen instabilities of internal magnetic-flux compactifications.

In Sect. 5, we demonstrate that supergravity effects can lead to large soft mass terms which stabilize most of these unstable vacua in certain regions of the parameter space. We also show that qualitative arguments based on cosmological constant cancellation generically require a large gravitino mass which, in a gravity-mediated supersymmetry breaking scenario, is consistent with the large soft terms that stabilize our unstable vacua. Finally, we conclude with comments about possible applications and generalizations of these constructions, and prospects for future research.

\section{Starting small: The Fayet-Iliopoulos model}

The simplest example of a model exhibiting a non-trivial, multiple-vacuum structure that we have in mind is the Fayet-Iliopoulos (FI) model of supersymmetry breaking [9]. Recall that this model has a single $U(1)$ gauge group with gauge coupling $g$ and FI coefficient $\xi$, two charged chiral superfields $\Phi^{( \pm)}$of charges \pm 1 , and a superpotential $W=m \Phi^{(+)} \Phi^{(-)}$. It turns out that the scalar potential for this model has three classes of extrema:

- Extrema with $v^{( \pm)} \equiv\left\langle\phi^{( \pm)}\right\rangle=0$. Such extremum solutions exist for all $\xi$, but represent stable vacua only for $m^{2} \pm g^{2} \xi \geq 0$.

- Extrema with $v^{(+)} \neq 0, v^{(-)}=0$. These solutions exist if $m^{2}+g^{2} \xi<0$, and are stable whenever they exist.

- Extrema with $v^{(-)} \neq 0, v^{(+)}=0$. These solutions exist if $m^{2}-g^{2} \xi<0$, and are stable whenever they exist.

Note that in the first class of solutions, both the gauge symmetry and $R$-symmetry of the model are unbroken, whereas in the second two classes of solutions they are both broken.* This vacuum structure is illustrated in Fig. 1, which indicates the appropriate vacuum as a function of $\xi$.

Of course, the FI model is actually defined by more than the single parameter $\xi$; we can just as easily consider $m$ as an additional defining parameter. We would

${ }^{*} D$-term breaking always preserves $R$-symmetries, independently of their detailed definition. By contrast, $F$-type breaking only breaks $R$-symmetries if the auxiliary field $F$ is charged under the $R$ symmetry. In all of the examples to be considered in this paper, we find that $F$-terms are charged under the $R$-symmetry if we define $R$-symmetry by requiring the superpotential to be invariant and assume that all matter fields have the same $R$-charge. Thus, $F$-breaking is equivalent to $R$ symmetry breaking. Indeed, for the FI model, if the superpotential charge is equal to 2 , then the matter superfield $R$-charges are 1 and therefore the $F$-terms have charges -1 . 


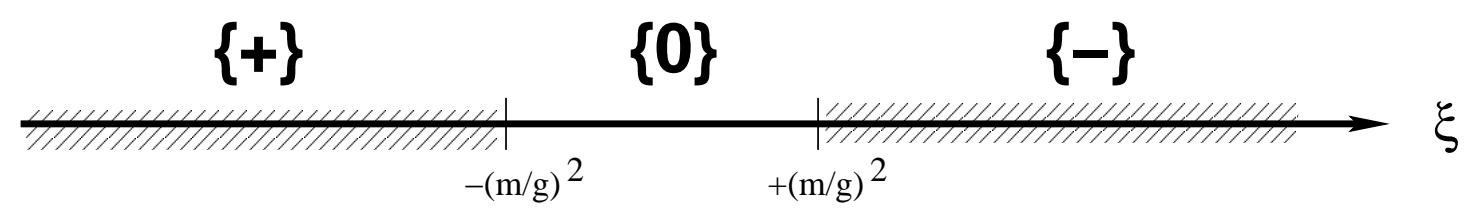

Figure 1: The vacuum structure of the Fayet-Iliopoulos (FI) model as a function of the FI coefficient $\xi$. The different classes of vacua outlined in the text (and here denoted $\{\emptyset\},\{+\}$, and $\{-\}$ respectively) occupy non-overlapping regions along the $\xi$-axis. In the regions corresponding to $\{+\}$ and $\{-\}$ vacua, we have indicated the existence of an unstable $\{\emptyset\}$ extremum in parenthesis. We have also shaded the regions corresponding to vacua with at least one non-zero vacuum expectation value, corresponding to vacua exhibiting $R$-symmetry breaking.

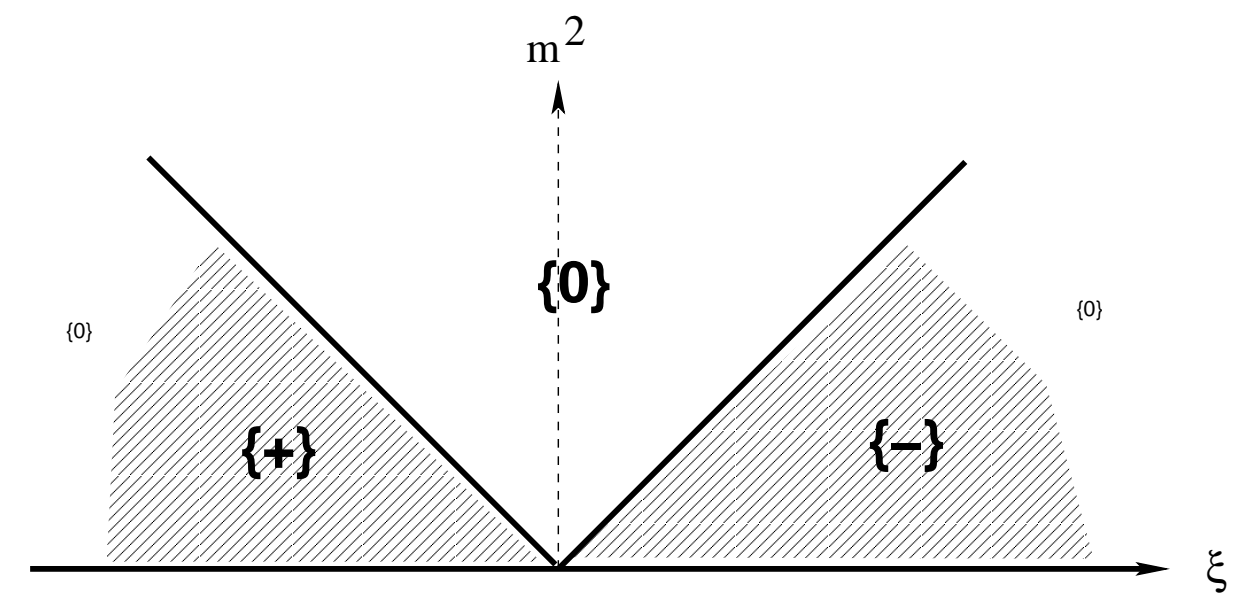

Figure 2: The vacuum structure of the FI model, now sketched as a function of $(\xi, m)$. Each region now has the shape of a pie-slice. The slopes of the diagonal lines are $\pm g^{2}$.

then sketch the vacuum structure of this model as in Fig. 2, which shows the vacuum structure as a function of both $\xi$ and $m$. The slopes of the diagonal lines are $\pm g^{2}$.

Finally, we may recognize that $g$ also represents an additional degree of freedom defining the model. In this case, it is relatively easy to visualize the resulting vacuum structure as a function of location in a three-dimensional space.

Suppose we were to ask a simple question: given the vacuum structure of this model, what is the net probability of randomly finding a vacuum that preserves $R$ symmetry? In this simple example, the answer clearly depends on the variables over which we average. If we consider $\xi$ to be the only variable as in Fig. 1 and perform our averaging with measure $d \xi$, we would claim that we have a virtual impossibility of realizing such a vacuum for any given (fixed) value of $(\mathrm{m} / \mathrm{g})^{2}$. This is because the $\{\emptyset\}$ region in Fig. 1 ultimately occupies only a region of finite size (set of measure 
zero) within the infinitely long $\xi$-axis. ${ }^{\dagger}$ On the other hand, if we consider both $\xi$ and $m$ to be our relevant variables as in Fig. 2 and calculate our average with measure $d \xi d m^{2}=2 m d \xi d m$, we would find that this probability is given by

$$
\operatorname{Prob}(\mathrm{R} \text { symm })=1-\frac{2}{\pi} \tan ^{-1} g^{2}
$$

Indeed, this is nothing but the ratio of the angles of the pie-slices in Fig. 2. Of course, this result would change if we were to take our measure to be, for example, $d \xi d m$ rather than $d \xi d m^{2}$. Finally, if we wish to consider $g$ as a third defining degree of freedom, we need to integrate the result in Eq. (2.1) over possible values of $g$. Clearly, if we use the measure $d g$, we would obtain a vanishing probability because of the infinite relative weighting given to vacua with large $g$. However, motivated by the pie-slice diagram in Fig. 2, we can define an angular variable $\theta_{g} \equiv \tan ^{-1} g^{2}$ and take our measure to be

$$
d \theta_{g}=\frac{1}{1+g^{4}} d g^{2} .
$$

This naturally suppresses the contributions from large- $g$ vacua, and yields the integrated result

$$
P \equiv \frac{2}{\pi} \int_{0}^{\pi / 2} d \theta_{g}\left(1-\frac{2}{\pi} \theta_{g}\right)=\frac{1}{2} .
$$

Of course, in practice we should perhaps only be integrating up to the limits of our perturbative treatment, restricting to $g \lesssim \sqrt{4 \pi}$, but this would introduce a possible strong dependence on the cutoff employed. Likewise, the measure in Eq. (2.2) is only one of many possible measures we could use. In any case, this discussion dramatically illustrates the importance of clearly defining both our relevant variables, our integration measure, and all associated cutoffs when averaging over multiple vacua.

It may seem strange to consider a coupling constant as a coordinate parametrizing a space of vacua. However, we recall that in string theory, the coupling constant is related to the vacuum expectation value (vev) of the dilaton, and such a vacuum expectation value is traditionally taken as one coordinate of an ensuing moduli space of vacua.

The issue of determining the appropriate landscape coordinates is clearly important, but unfortunately model-dependent. In the popular compactifications with fluxes [6], the gauge couplings on D3-branes depend on the dilaton which, in turn, depends on the fluxes. However, since we ultimately have to restrict our attention to gauge couplings at high scales which are compatible with the experimentally measured couplings at low scales, these couplings should be $\sim \mathcal{O}(1)$. A pragmatic attitude

\footnotetext{
${ }^{\dagger}$ In practice, of course, we must have $\xi \lesssim M_{P}^{2}$ where $M_{P}$ is the Planck scale, so our parameter space is not truly infinite in extent. Also, the FI coefficients $\xi$ are typically quantized. Throughout this paper, however, we shall consider the continuous limit and imagine that our parameter spaces are infinite in extent.
} 
would then be to focus directly on that subset of fluxes leading to realistic gauge couplings. By contrast, the FI terms depend on moduli fields and charge assignments; they are certainly landscape-dependent and are therefore natural landscape coordinates. The supersymmetric mass in the FI model can also be generated by suitable fluxes, in analogy with the $\mu$-term of MSSM [10]. Likewise, the supergravity-induced soft mass terms that we shall introduce in subsequent sections are also flux-dependent. Thus, we see that essentially all mass-like parameters are natural landscape coordinates.

An important constraint is that the FI terms and supersymmetric and soft mass terms are bounded in any string compactifications. Indeed, FI terms are proportional to vev's of various moduli fields and to the sum of $U(1)$ charges, and are always smaller than the string and the Planck scale. Likewise, the various fluxes must generically satisfy Ramond-Ramond tadpole constraints; in most cases, these constraints place upper limits on their possible values. Thus, in Figs. 1 and 2 (as well as all subsequent parameter-space diagrams in this paper), we should implicitly impose a cutoff on the FI terms and various mass parameters. This cutoff is model-dependent, but should roughly be of size $\sim \mathcal{O}\left(M_{P}\right)$ where $M_{P}$ is the Planck mass.

An interesting result of our analysis of the FI model in Fig. 2 is that the vacuum structure is energy-dependent. Indeed, if $Z_{m}(E)$ denotes the wavefunction renormalization factor for the matter fields (which we take to be equal for the two superfields) and $Z(E)$ denotes the wavefunction renormalization factor for the gauge superfield, then the boundaries between the $\{+\}$ and $\{\emptyset\}$ regions and the $\{\emptyset\}$ and $\{-\}$ regions will scale according to the energy-dependent expressions $\pm g^{2} Z_{m}(E) / Z(E)$. These boundaries therefore rotate under the renormalization group. This opens up the possibility of having a vacuum which passes from one region to another under RG flow, a phenomenon which can generate phase transitions with potentially interesting cosmological implications. We will study this phenomenon in more detail in Sect. 3, within the framework of a more interesting (and also slightly more complicated) example.

\section{Constructing a toy model}

It turns out that the FI model is not sufficiently complex for our purposes. In this section, we shall therefore turn to the next-simplest model which, as we shall see, will exhibit almost all of the relevant features which we shall encounter when we proceed to consider more complicated situations in subsequent sections. In particular, we shall see that this toy model gives rise to a non-trivial "landscape" consisting of multiple stable (or metastable) vacua with different low-energy phenomenologies, unstable extrema, phase transitions, etc. Moreover, even though this toy model is relatively simple, we expect that the resulting landscape is literally a component of the full string-theory landscape in cases of string models with multiple $U(1)$ gauge factors.

Our model consists of two $U(1)$ gauge symmetries, denoted $U(1)_{1}$ and $U(1)_{2}$, and three $\mathcal{N}=1$ chiral superfields, $\Phi_{i=1,2,3}$. The charges of these chiral superfields under 


\begin{tabular}{||c||cc||}
\hline \hline & $U(1)_{1}$ & $U(1)_{2}$ \\
\hline \hline$\Phi_{1}$ & -1 & 0 \\
$\Phi_{2}$ & 1 & -1 \\
$\Phi_{3}$ & 0 & 1 \\
\hline \hline
\end{tabular}

Table 1: $U(1)$ charge assignments for chiral superfields in our toy model.

the $U(1)$ gauge symmetries are chosen as in Table 1 . In a string-theory context, such $U(1)$ gauge factors can be imagined as arising from different D-branes, and the $\Phi_{i}$ fields can arise as strings stretched between these branes. We shall also assume that the $\mathcal{N}=1$ supersymmetry is broken by Fayet-Iliopoulos $D$-term coefficients $\xi_{1}$ and $\xi_{2}$, and by a renormalizable Wilson-line superpotential of the form

$$
W=\lambda \Phi_{1} \Phi_{2} \Phi_{3}
$$

Our model is thus defined by three parameters, $\left\{\xi_{1}, \xi_{2}, \lambda\right\}$, and our goal will be to study the vacuum structure of this model as a function of these parameters. Of course, the resulting physics is unchanged if $\lambda \rightarrow-\lambda$. We shall therefore restrict ourselves to $\lambda \geq 0$ for simplicity.

It is straightforward to analyze the vacuum states of this theory. As usual, the scalar potential is given by

$$
V=\frac{1}{2} \sum_{a} g_{a}^{2} D_{a}^{2}+\sum_{i}\left|F_{i}\right|^{2}
$$

where the $D$ - and $F$-terms are given by

$$
D_{a}=\sum_{i} q_{i}^{(a)}\left|\phi_{i}\right|^{2}+\xi_{a}, \quad F_{i}=\frac{\partial W}{\partial \phi_{i}} .
$$

Here $a=1,2$ is the index of the gauge group $U(1)$ factor, $g_{a}$ is the gauge coupling corresponding to the $U(1)_{a}$ factor, and $i=1,2,3$ is the index of the chiral superfield. Thus, $q_{i}^{(a)}$ denotes the $U(1)_{a}$ charge of $\Phi_{i}$. In most of our considerations the gauge couplings $g_{a}$ will not be important, so we will henceforth consider $g_{1}=g_{2}=1$ for simplicity. We will reinstall gauge couplings whenever relevant for our analysis. Our task is to determine the extrema of $V$ by seeking solutions to the simultaneous equations

$$
\frac{\partial V}{\partial \phi_{i}}=\frac{\partial V}{\partial \phi_{i}^{*}}=0
$$

and then to determine whether these extrema represent stable (or metastable) vacua by calculating the eigenvalues of the corresponding mass matrix

$$
\mathcal{M}^{2} \equiv\left(\begin{array}{cc}
\frac{\partial^{2} V}{\partial \phi_{i}^{*} \partial \phi_{j}} & \frac{\partial^{2} V}{\partial \phi_{i}^{*} \partial \phi_{j}^{*}} \\
\frac{\partial^{2} V}{\partial \phi_{i} \partial \phi_{j}} & \frac{\partial^{2} V}{\partial \phi_{i} \partial \phi_{j}^{*}}
\end{array}\right)
$$


evaluated at the extrema. Note that in general, there will be a zero eigenvalue for each spontaneously broken $U(1)$; these eigenvalues correspond to the resulting Nambu-Goldstone bosons. The extrema defined by Eq. (3.4) represent stable (or metastable) vacua only if each of the remaining eigenvalues is positive.

Just as with the FI model in Sect. 2, it will prove convenient to group the extrema of $V$ into classes depending on which combinations of chiral superfields receive nonzero vacuum expectation values. This classification will help in determining such features as whether the sources of SUSY-breaking are primarily $D$-terms or $F$-terms, and whether they are likely to lead to $R$-symmetry breaking when incorporated into a supergravity framework. Since there are three chiral superfields in this example, there are correspondingly $8=2^{3}$ classes of extrema of $V$. Denoting $\left\langle\Phi_{i}\right\rangle \equiv v_{i}$, we shall define our classes of extrema according to their values of $v_{i}$, using the notation $\left\{\right.$ ijk...\} to indicate the class of vacua in which $v_{i}, v_{j}, v_{k}, \ldots$ are all non-zero (with $\{\emptyset\}$ denoting the vacua in which all $v_{i}$ vanish). Note that in this toy model, we can choose all $v_{i}$ to be real without loss of generality.

\section{$3.1 \lambda=1$}

Let us first consider the vacuum structure of this model when $\lambda=1$. Our results are then as follows. We find that $\{\emptyset\}$ extrema exist for all values of $\left(\xi_{1}, \xi_{2}\right)$, but are always unstable. This result, of course, is intuitively obvious, since $\{\emptyset\}$ vacua are essentially at the unstable center of the SUSY-breaking "Mexican hat". By contrast, $\{1\}$ extrema exist only for $\xi_{1}>0$. However, these extrema are stable only within the subregion defined by $\left|\xi_{2}\right|<\xi_{1}$. Likewise, $\{\mathbf{2}\}$ extrema exist for $\xi_{2}>\xi_{1}$ and are stable in the region $\xi_{1}<0, \xi_{2}>0$, while $\{\mathbf{3}\}$ extrema exist only for $\xi_{2}<0$ and are stable within the subregion $\left|\xi_{1}\right|<\left|\xi_{2}\right|$. Similarly, $\{\mathbf{1 2}\}$ extrema exist only for $0<\xi_{1}<\xi_{2}$ and are stable everywhere within this region, while $\{23\}$ extrema exist only for $\xi_{1}<\xi_{2}<0$ and are also stable everywhere within this region. Finally, for $\lambda=1$, extrema in Classes $\{\mathbf{1 3}\}$ and $\{\mathbf{1 2 3}\}$ do not exist for any values of $\left(\xi_{1}, \xi_{2}\right)$.

It is clear from these results that for $\lambda=1$, there are no regions in $\left(\xi_{1}, \xi_{2}\right)$ parameter space in which there are multiple stable vacua. Instead, the different classes of vacua occupy non-overlapping regions which completely fill out this space. This situation is sketched in Fig. 3.

It is important to interpret the diagram in Fig. 3 correctly. Each point in the $\left(\xi_{1}, \xi_{2}\right)$ plane represents a unique theory, i.e., a unique model parametrized by its defining parameters $\xi_{1}$ and $\xi_{2}$. Thus, this plane is nothing but a field-theoretic "landscape" for this two- $U(1)$ theory with three chiral superfields. For each point in the landscape, there is a unique vacuum whose energy can be calculated from Eq. (3.2). For example, for models within the region specified by $\xi_{2}>-\xi_{1}$ and $\xi_{2}<0$ (i.e., the lower half of the $\{\mathbf{1}\}$ region in Fig. 3 ), the energy of the stable $\{\mathbf{1}\}$ extremum is given by $V_{\{\mathbf{1}\}}=\xi_{2}^{2} / 2$. This is to be contrasted with the unstable $\{\emptyset\}$ and $\{3\}$ extrema which also exist for models in this region, with energies given by 


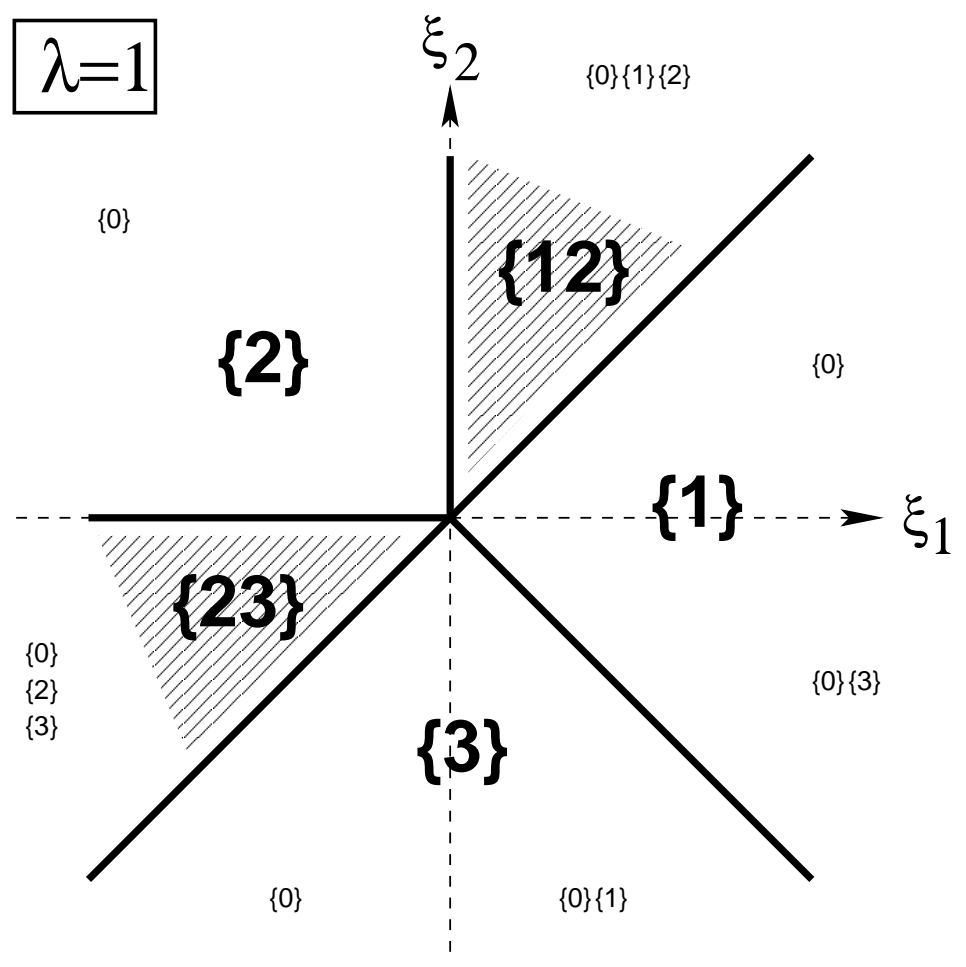

Figure 3: The "landscape" of our toy model with two $U(1)$ gauge fields and three chiral superfields, sketched for $\lambda=1$. The different classes of vacua (labelled according to which chiral fields receive non-zero vacuum expectation values) occupy the non-overlapping regions indicated above in bold type, while unstable extrema in each region are indicated in smaller type. We have shaded the regions corresponding to vacua with two non-zero vacuum expectation values (Classes $\{\mathbf{1 2}\}$ and $\{\mathbf{2 3}\}$ ); these are regions in which $R$-symmetry is potentially broken.

$V_{\{\emptyset\}}=\left(\xi_{1}^{2}+\xi_{2}^{2}\right) / 2$ and $V_{\{\mathbf{3}\}}=\xi_{1}^{2} / 2$ respectively. Note that indeed $V_{\{\mathbf{1}\}}<V_{\{\emptyset\},\{\mathbf{3}\}}$.

At this stage, we can already reach an interesting conclusion: for the $\lambda=1$ landscape, the average ratio of stable extrema relative to all extrema is only

$$
\begin{aligned}
\text { Probability that extremum is stable } & =\frac{1}{8}\left(\frac{1}{4}+\frac{1}{2}+\frac{1}{3}+\frac{1}{3}+\frac{1}{2}+\frac{1}{4}+\frac{1}{2}+\frac{1}{2}\right) \\
& =\frac{19}{48} \approx 0.396 .
\end{aligned}
$$

Note that in calculating this probability, we are averaging over the landscape with a uniform measure (averaging the stability probabilities in each of the eight octants of the plane), imagining that all values of our landscape coordinates $\left(\xi_{1}, \xi_{2}\right)$ are equally likely. This result is significantly larger than the value $(1 / 2)^{6}$ which we might have naïvely expected from the fact that our mass matrix in Eq. (3.5) has six eigenvalues, any one of which randomly could have been negative. Even if we allow for potentially 
as many as two zero eigenvalues corresponding to Nambu-Goldstone bosons for the two $U(1)$ gauge factors in this toy model, we still have a much higher probability of finding stable extrema than expected.

In a similar vein, we can also see from Fig. 3 that a randomly chosen model in the landscape has exactly a $25 \%$ chance of breaking $R$-symmetry when $\lambda=1$. Those regions populated by $R$-symmetry breaking vacua are shaded in Fig. 3 .

In this landscape, there do not exist any regions with overlapping stable vacua. In other words, there are no values of $\left(\xi_{1}, \xi_{2}\right)$ giving rise to multiple stable vacua. Thus, each vacuum is absolutely stable with respect to dynamics within that model. However, these different vacua fill out a landscape in theory space, as sketched in Fig. 3, and these vacua ultimately have different energies which depend on the landscape coordinates $\left(\xi_{1}, \xi_{2}\right)$. For $\lambda=1$, the different classes of extrema have energies:

$$
\begin{aligned}
V_{\{\emptyset\}} & =\frac{1}{2}\left(\xi_{1}^{2}+\xi_{2}^{2}\right)=V_{\{\mathbf{1}\}}+V_{\{\mathbf{3}\}} \\
V_{\{\mathbf{1}\}} & =\frac{1}{2} \xi_{2}^{2} \\
V_{\{\mathbf{2}\}} & =\frac{1}{4}\left(\xi_{1}+\xi_{2}\right)^{2} \\
V_{\{\mathbf{3}\}} & =\frac{1}{2} \xi_{1}^{2} \\
V_{\{\mathbf{1 2}\}} & =\frac{1}{4}\left(-\xi_{1}^{2}+2 \xi_{1} \xi_{2}+\xi_{2}^{2}\right)=V_{\{\mathbf{2}\}}-V_{\{\mathbf{3}\}} \\
V_{\{\mathbf{2 3}\}} & =\frac{1}{4}\left(\xi_{1}^{2}+2 \xi_{1} \xi_{2}-\xi_{2}^{2}\right)=V_{\{\mathbf{2}\}}-V_{\{\mathbf{1}\}} .
\end{aligned}
$$

Moreover, note from Eq. (3.7) that Class $\{\mathbf{1}\}$ vacua with $\xi_{2}=0$, Class $\{\mathbf{2}\}$ vacua with $\xi_{2}=-\xi_{1}$, and Class $\{3\}$ vacua with $\xi_{1}=0$ are all SUSY-preserving, with $V=0$. Even though these SUSY-preserving vacua only form a one-dimensional space of vacua within the larger two-dimensional landscapes we have plotted (thereby essentially forming a set of measure zero within the full landscape), these vacua represent the lowest-energy vacua within their respective regions. However, unless transitions between different vacua in the landscape are somehow possible, the existence of such supersymmetric vacua at one landscape location does not destabilize the non-supersymmetric vacua that may exist at other landscape locations.

We see, then, that this "landscape" is indeed a very realistic, and ultimately completely calculable, model of the string-theoretic landscape. Indeed, we have a space of models, with each parametrized by the model-defining parameters $\left(\xi_{1}, \xi_{2}\right)$, and each with a different vacuum and vacuum energy.

\section{$3.2 \lambda<1$}

It is important to consider how this landscape picture is modified for $\lambda \neq 1$. Considering first the case with $\lambda<1$, we find that Class $\{\emptyset\}$ extrema continue to exist for all $\left(\xi_{1}, \xi_{2}\right)$ and continue to be unstable everywhere. Class $\{\mathbf{1}\}$ extrema, by contrast, continue to exist for all $\xi_{1}>0$, but are now stable only within the smaller region $\left|\xi_{2}\right|<\lambda^{2} \xi_{1}$. Similarly, Class $\{2\}$ extrema continue to exist for all $\xi_{2}>\xi_{1}$ and 
are stable only within the region defined by

$$
\left(1-\lambda^{2}\right) \xi_{2}<-\left(\lambda^{2}+1\right) \xi_{1}, \quad\left(\lambda^{2}+1\right) \xi_{2}>\left(\lambda^{2}-1\right) \xi_{1}
$$

while Class $\{\mathbf{3}\}$ extrema continue to exist for all $\xi_{2}<0$ but are stable only within the smaller region $\left|\xi_{1}\right|<\lambda^{2}\left|\xi_{2}\right|$. All of these results reduce to the previous case as $\lambda \rightarrow 1$. In general, these results indicate that the $\{\mathbf{1}\},\{\mathbf{2}\}$, and $\{\mathbf{3}\}$ regions become smaller as $\lambda \rightarrow 0$, occupying narrower and narrower "pie-slices" in the $\left(\xi_{1}, \xi_{2}\right)$ landscape plane. Specifically, each of these "pie-slices" has total angle $\theta_{\{\mathbf{1}\},\{\mathbf{2}\},\{\mathbf{3}\}}=2 \theta_{\lambda}$, where

$$
\theta_{\lambda} \equiv \tan ^{-1} \lambda^{2}
$$

and differ only in their orientations in the $\left(\xi_{1}, \xi_{2}\right)$ plane, with the Class $\{1\}$ pie-slice centered around the positive $\xi_{1}$-axis, the Class $\{2\}$ pie-slice centered around the $\xi_{2}=-\xi_{1}>0$ diagonal axis, and the Class $\{3\}$ pie-slice centered around the negative $\xi_{2}$-axis. Thus, as $\lambda \rightarrow 0$, the $\{\mathbf{1}\},\{\mathbf{2}\}$, and $\{\mathbf{3}\}$ regions disappear entirely. A sketch of these results for $\lambda=1 / 2$ is shown in Fig. $4(\mathrm{a})$.
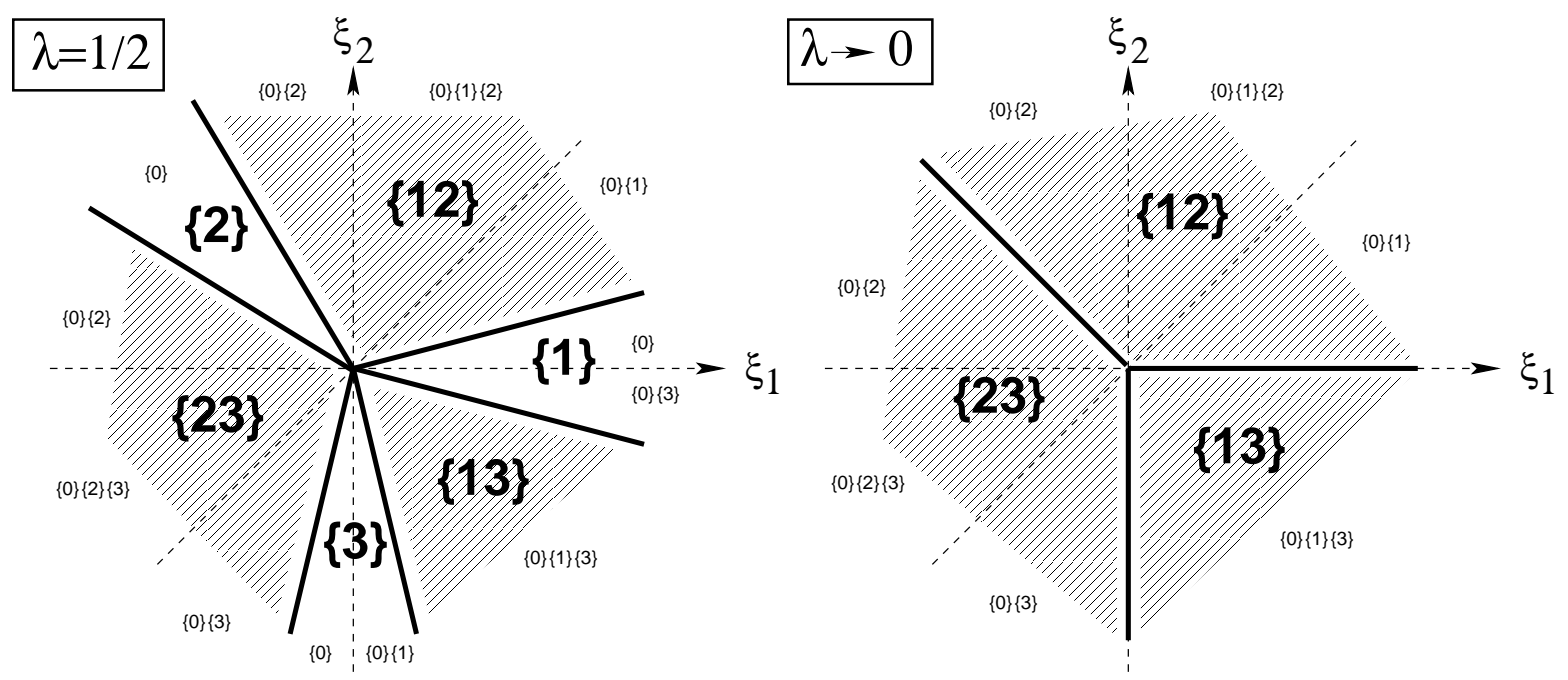

Figure 4: (a) Left figure: Same landscape as in Fig. 3, now sketched for $\lambda=1 / 2$. The $\{\mathbf{1}\}$, $\{\mathbf{2}\}$, and $\{\mathbf{3}\}$ regions have each become smaller, remaining equal in size, while the $\{\mathbf{1 2}\}$ and $\{\mathbf{2 3}\}$ regions (as well as the new $\{\mathbf{1 3}\}$ region) have expanded to fill in the gaps. Just as for $\lambda=1$, the different classes of vacua occupy non-overlapping regions in this landscape, and we have indicated the unstable extrema in parentheses. (b) Right figure: As $\lambda \rightarrow 0$, the $\{\mathbf{1}\},\{\mathbf{2}\}$, and $\{\mathbf{3}\}$ regions disappear entirely, leaving a landscape entirely dominated by non-overlapping $\{\mathbf{1 2}\},\{\mathbf{2 3}\}$, and $\{\mathbf{1 3}\}$ regions. These regions ultimately represent flat directions in the $\lambda=0$ limit.

Just as in the $\lambda=1$ case, extrema in Classes $\{\mathbf{1 2}\}$ and $\{23\}$ continue to exist in the gaps between the $\{\mathbf{1}\}$ and $\{\mathbf{2}\}$ regions, and $\{\mathbf{2}\}$ and $\{3\}$ regions, respectively. As evident from Fig. 4(a), each of these regions has angle $\theta_{\{\mathbf{1 2}\},\{\mathbf{2 3}\}}=3 \pi / 4-2 \theta_{\lambda}$. 
Moreover, these extrema are stable everywhere within these regions. Thus, as $\lambda \rightarrow 0$, these regions become larger rather than smaller. The new feature for $\lambda<1$, however, is the emergence of new Class $\{\mathbf{1 3}\}$ extrema which populate the gap that has opened up between the $\{\mathbf{1}\}$ and $\{\mathbf{3}\}$ regions, with angle $\theta_{\{\mathbf{1 3}\}}=\pi / 2-2 \theta_{\lambda}$. Just as with the extrema in Classes $\{\mathbf{1 2}\}$ and $\{\mathbf{2 3}\}$, extrema in Class $\{\mathbf{1 3}\}$ are stable wherever they exist. There continue to be no extrema, stable or unstable, in Class $\{123\}$ for $\lambda>0$.

We see, then, that just as for $\lambda=1$, the different classes of vacua continue to occupy non-overlapping regions for $\lambda<1$. Indeed, as $\lambda \rightarrow 0$, the $\{1\},\{2\}$, and $\{3\}$ regions disappear entirely, leaving a landscape entirely dominated by nonoverlapping $\{\mathbf{1 2}\},\{\mathbf{1 3}\}$, and $\{\mathbf{2 3}\}$ regions, with angles $\theta_{\{\mathbf{1 2}\},\{\mathbf{2 3}\}}=3 \pi / 4$ and $\theta_{\{\mathbf{1 3}\}}=$ $\pi / 2$ respectively. This situation is illustrated in Fig. 4(b).

The $\lambda=0$ limit is somewhat special. In this limit, of course, we find that the three regions in Fig. 4(b) continue to exist. However, rather than representing stable vacua, each of these regions now represents a class of flat directions. This makes sense, since SUSY is unbroken for these extrema in the $\lambda=0$ limit, even for arbitrary values of the FI coefficients $\xi_{i}$. Likewise, $R$-symmetry is preserved in these regions. Of course, the unstable extrema listed in Fig. 4(b) continue to represent solutions with broken SUSY and positive vacuum energies, even when $\lambda=0$.

It is easy to interpret this flat direction in terms of the vacuum expectation values $v_{i}$. Recall that for any $\lambda>0$, there are no extrema in the $\{\mathbf{1 2 3}\}$ class. However, for $\lambda=0$, a new $\{\mathbf{1 2 3}\}$ extremum appears, corresponding to the general solution

$$
\left|v_{1}\right|^{2}=\left|v_{2}\right|^{2}+\xi_{1}, \quad\left|v_{3}\right|^{2}=\left|v_{2}\right|^{2}-\xi_{2} .
$$

These equations have solutions for $\left(v_{1}, v_{2}, v_{3}\right)$ for all $\left(\xi_{1}, \xi_{2}\right)$, and thus can be considered to exist throughout the entire $\left(\xi_{1}, \xi_{2}\right)$ landscape. They are also SUSY-preserving, maintaining $V=0$ for all choices of $\left|v_{2}\right|^{2}$ in Eq. (3.10), and serve as the algebraic representation of the flat direction when $\lambda=0$. Indeed, depending on the value of $\left(\xi_{1}, \xi_{2}\right)$, the corresponding $\{\mathbf{1 2}\},\{\mathbf{1 3}\}$, or $\{\mathbf{2 3}\}$ solution that appears in Fig. $4(\mathrm{~b})$ is only a special case of the more general $\{\mathbf{1 2 3}\}$ solution in Eq. (3.10). For example, the $\{12\}$ region in Fig. 4(b), which corresponds to solutions with $\left|v_{1}\right|^{2}=\xi_{1}+\xi_{2}$, $\left|v_{2}\right|^{2}=\xi_{2}$, and $\left|v_{3}\right|^{2}=0$, can be viewed as a special case of Eq. (3.10) with $\left|v_{2}\right|^{2}=\xi_{2}$. Thus, when $\lambda=0$, we should more correctly state that the entire $\left(\xi_{1}, \xi_{2}\right)$ landscape can be described as a single flat direction corresponding to the $\{\mathbf{1 2 3}\}$ solution in Eq. (3.10).

\section{$3.3 \lambda>1$}

Let us now turn to the situation when $\lambda>1$. In such cases, the above algebraic conditions for the existence and stability of the $\{\emptyset\},\{\mathbf{1}\},\{\mathbf{2}\}$, and $\{\mathbf{3}\}$ extrema do not change. Thus, as $\lambda$ grows beyond 1, we see that the pie-slices occupied by stable extrema in these classes actually grow rather than shrink, with angles still given by 
$\theta_{\{\mathbf{1}\},\{\mathbf{2}\},\{\mathbf{3}\}}=2 \theta_{\lambda}=2 \tan ^{-1} \lambda^{2}$. Indeed, as $\lambda \rightarrow \infty$, each of these stable subregions expands to fill its corresponding existence region, so that all extrema in these classes become stable as $\lambda \rightarrow \infty$.

Given these results, we see that Regions $\{1\}$ and $\{3\}$ actually overlap for $\lambda>1$, with overlap angle $\theta_{\{\mathbf{1}\}+\{\mathbf{3}\}}=2 \theta_{\lambda}-\pi / 2$. Likewise, the $\{\mathbf{2}\}$ region separately overlaps with the $\{1\}$ region and with the $\{3\}$ region for $\lambda>\lambda_{*}$, where

$$
\lambda_{*} \equiv(1+\sqrt{2})^{1 / 2} \approx 1.55 \quad \Longleftrightarrow \quad \theta_{*} \equiv \tan ^{-1} \lambda_{*}^{2}=\frac{3 \pi}{8} .
$$

In the latter case, these overlap regions have angles $\theta_{\{\mathbf{1}\}+\{\mathbf{2}\}}=\theta_{\{\mathbf{2}\}+\{\mathbf{3}\}}=2 \theta_{\lambda}-3 \pi / 4=$ $2\left(\theta_{\lambda}-\theta_{*}\right)$. Thus, we see that we now have cases in which a single point in the landscape can give rise to multiple (meta)stable extrema. Of course, only the extremum with the lowest energy in each case is truly stable within the specified model, while the remaining extremum is only metastable. In this example, we find from Eq. (3.7) that the $\{1\}$ vacuum is truly stable for $\xi_{2}<\xi_{1}$, while the $\{3\}$ vacuum is truly stable for $\xi_{1}<\xi_{2}$. This conclusion holds for all $\lambda>1$.

We stress that this metastability exists within a single point in the landscape (i.e., within a single model characterized by specific values of $\xi_{1}$ and $\xi_{2}$ ). This is quite different from instabilities across different models at different landscape locations; such theory-to-theory transitions are beyond the framework of quantum field theory, but have been conjectured to occur in string theory. If such transitions can occur in string theory, then we can expect the full dynamics on such a landscape to involve both intra-model as well as inter-model metastability. However, there is no evidence that such inter-model transitions can occur.

An important question is to determine the fate of the $\{\mathbf{1 2}\},\{\mathbf{1 3}\}$, and $\{\mathbf{2 3}\}$ extrema for $\lambda>1$. For $1<\lambda<\lambda_{*}$, extrema in the $\{\mathbf{1 2}\}$ and $\{23\}$ classes continue to exist within their remaining gap regions and are always stable, with angles $\theta_{\{\mathbf{1 2}\},\{\mathbf{2 3}\}}=$ $3 \pi / 4-2 \theta_{\lambda}$. Moreover, for all $\lambda>1,\{13\}$ extrema actually re-emerge within the Class $\{\mathbf{1}\}+\{\mathbf{3}\}$ overlap region, but are now unstable. As always in this toy model, there are no extrema in Class $\{\mathbf{1 2 3}\}$ except at $\lambda=0$. We thus obtain a landscape as sketched in Fig. 5.

The final remaining case corresponds to $\lambda>\lambda_{*}$. For $\lambda$ in this range, the $\{1\}$, $\{2\}$, and $\{3\}$ regions all mutually overlap in a pairwise fashion. (Note that these three regions never have a simultaneous common overlap.) In such cases, we then find that extrema in Classes $\{12\},\{13\}$, and $\{23\}$ all continue to exist in their respective overlap regions, but that all of these extrema are now unstable. We thus obtain a landscape as sketched in Fig. 6(a). Note that as $\lambda \rightarrow \infty$, our landscape reduces to that sketched in Fig. 6(b). 


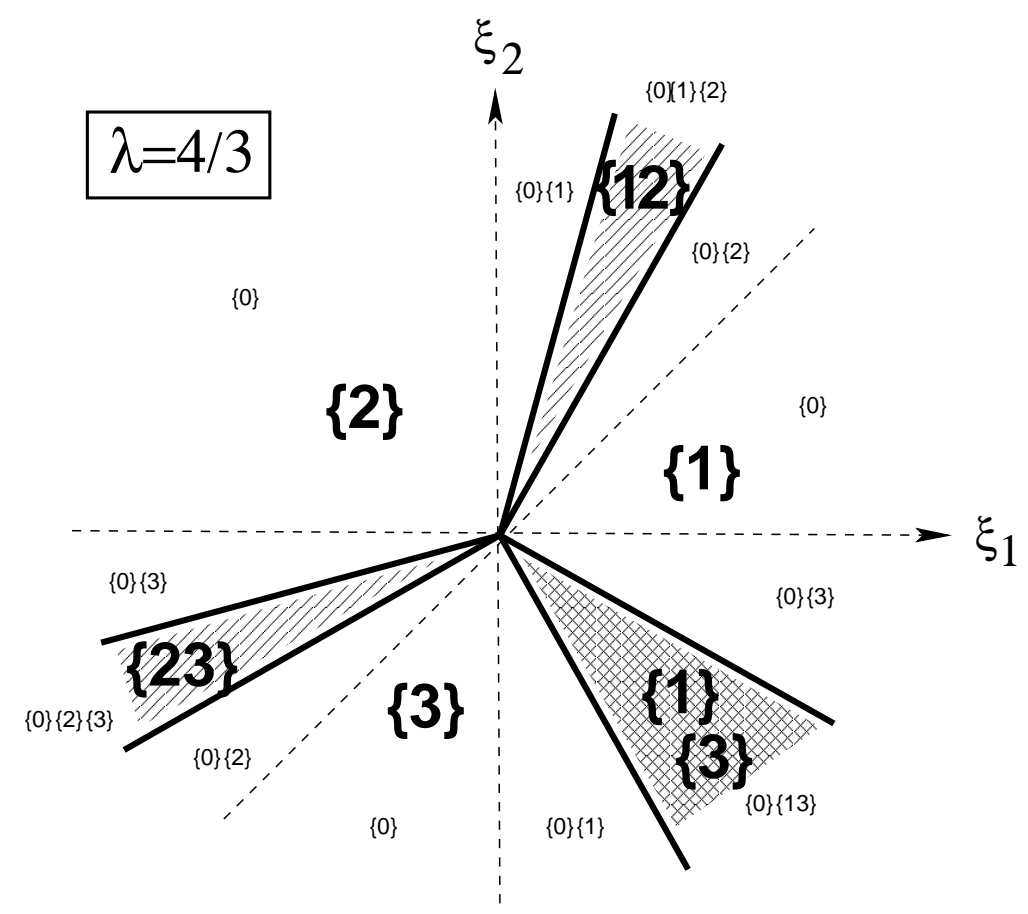

Figure 5: Same landscape as in previous figures, now sketched for $\lambda=4 / 3$. Regions $\{\mathbf{1}\}$, $\{\mathbf{2}\}$, and $\{\mathbf{3}\}$ have each become larger, with a new overlap region (indicated with double cross-hatching) between the $\{\mathbf{1}\}$ and $\{\mathbf{3}\}$ regions. Class $\{\mathbf{1 3}\}$ extrema exist but are now unstable in this overlap region, while stable Class $\{\mathbf{1 2}\}$ and $\{\mathbf{2 3}\}$ vacua continue to populate the gaps between Regions $\{\mathbf{1}\}$ and $\{\mathbf{2}\}$, and Regions $\{\mathbf{2}\}$ and $\{\mathbf{3}\}$, respectively. There continue to be no overlaps amongst Regions $\{1\} /\{12\} /\{2\}$ or Regions $\{2\} /\{23\} /\{3\}$.

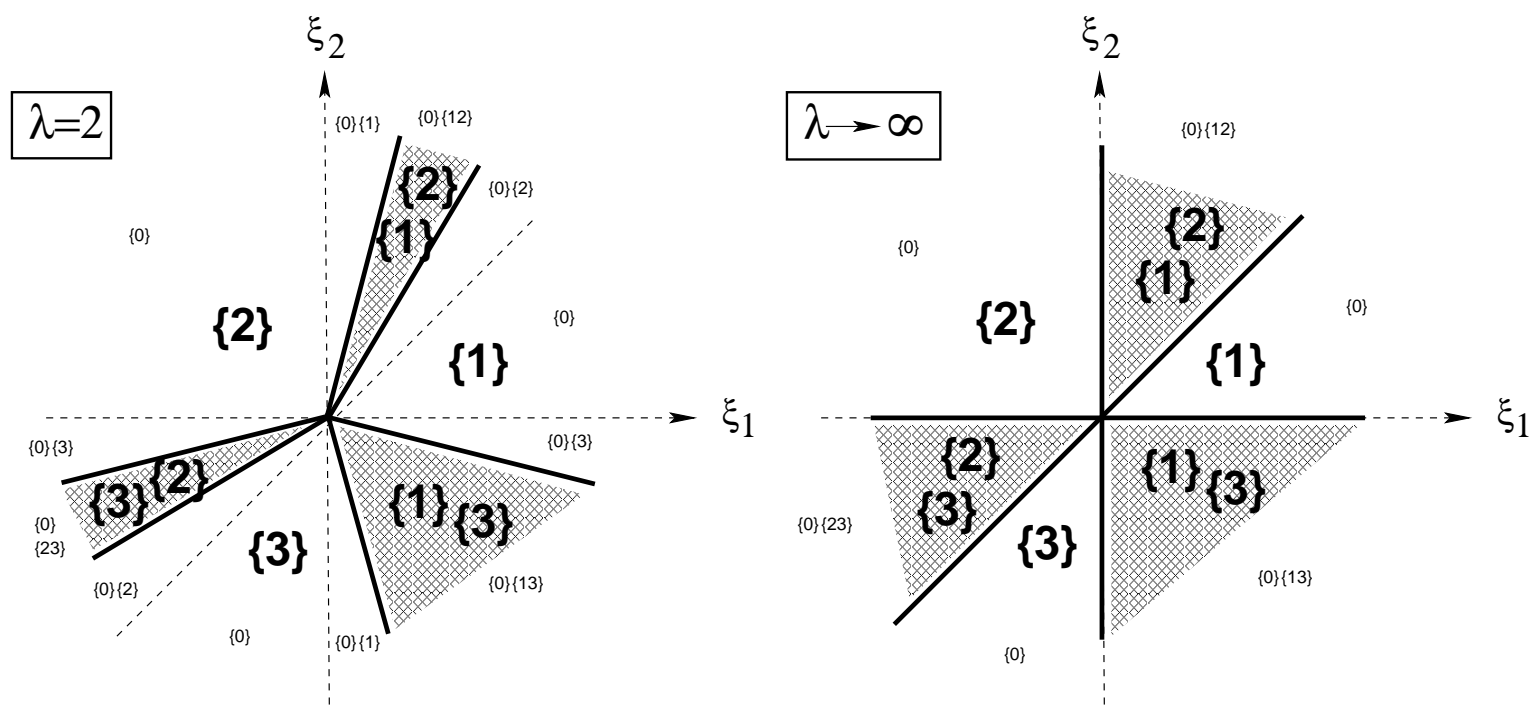

Figure 6: Same landscape as in previous figures, now sketched (a) for $\lambda=2$ (left), and (b) as $\lambda \rightarrow \infty$ (right). Since $\lambda>\lambda_{*}$ in each case, the $\{\mathbf{1}\},\{\mathbf{2}\}$, and $\{\mathbf{3}\}$ regions all mutually overlap; these three overlap regions are indicated with double cross-hatching. Extrema in Classes $\{\mathbf{1 2}\},\{\mathbf{1 3}\}$, and $\{\mathbf{2 3}\}$ exist in these overlap regions, but are always unstable for $\lambda>\lambda_{*}$. 


\subsection{Landscape probabilities and integration measures}

Just as in the $\lambda=1$ case, we can calculate the fraction of stable extrema relative to all extrema as a function of $\lambda$; we merely evaluate this fraction in each relevant pie-slice, and then properly weight these pie-slices according to their opening angles. It turns out that as a function of $\lambda$, this fraction is given by

$$
\text { Prob }(\text { stable } \lambda)= \begin{cases}\left(7 \pi+10 \tan ^{-1} \lambda^{2}\right) / 24 \pi & \text { for } \lambda \leq 1, \\ \left(15 \pi+16 \tan ^{-1} \lambda^{2}\right) / 48 \pi & \text { for } 1 \leq \lambda \leq \lambda_{*}, \\ \left(\pi+2 \tan ^{-1} \lambda^{2}\right) / 4 \pi & \text { for } \lambda \geq \lambda_{*} .\end{cases}
$$

Note that this probability agrees with Eq. (3.6) when $\lambda=1$, and generally increases monotonically from $7 / 24 \approx 0.29$ at $\lambda=0$ to exactly $1 / 2$ at $\lambda \rightarrow \infty$. For example, it is straightforward to verify from Fig. 6 (b) that this fraction is indeed $1 / 2$ in the $\lambda \rightarrow \infty$ limit. A plot of this probability as a function of $\lambda$ is shown in Fig. 7. Once again, we stress that we are averaging over our $\left(\xi_{1}, \xi_{2}\right)$ landscape with a uniform measure when calculating this probability. As already noted below Eq. (3.6), we see that this probability is significantly greater for all values of $\lambda$ than we might have naïvely expected.

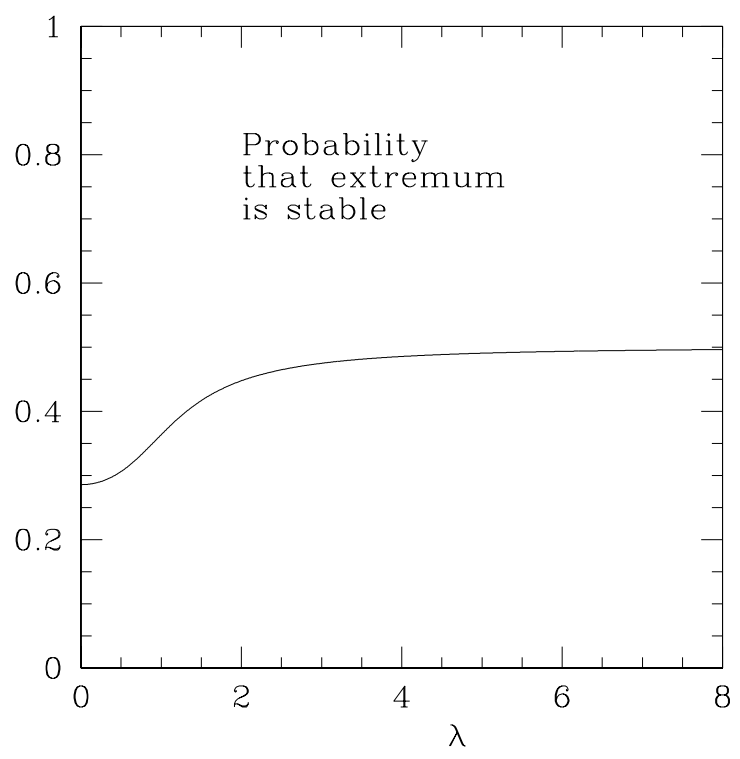

Figure 7: The probability that a given extremum in our toy model is stable, plotted as a function of the Yukawa coupling $\lambda$ and integrated over all $\left(\xi_{1}, \xi_{2}\right)$. This function increases monotonically from $7 / 24$ when $\lambda=0$, and asymptotes to $1 / 2$ as $\lambda \rightarrow \infty$. Note that in all cases, this probability is significantly greater than we would have naïvely expected based on a random assignment of signs for the eigenvalues of the six-dimensional mass matrix.

Of course, $\lambda$ is actually a landscape coordinate; just as with $\xi_{1}$ and $\xi_{2}$, the value 
of the parameter $\lambda$ specifies a particular model under study. ${ }^{\ddagger}$ In other words, as discussed at the beginning of this section, our toy model really has a three-dimensional landscape, with these figures showing only particular fixed- $\lambda$ slices through this landscape. Thus, in calculating the probability of finding a stable extremum from amongst all extrema, we should really be averaging over $\lambda$ (our third direction) as well.

However, this illustrates another critical issue for the landscape. What measure should we use when performing our $\lambda$-integration? If we were to perform our averaging process using the measure $d \lambda$, we would find that the resulting average probability would be close to $1 / 2$ because of the overwhelming dominance of the contributions from large $|\lambda|$. This suggests that the simple measure $d \lambda$ may not be the most appropriate measure for the Yukawa coupling $\lambda$, even with an appropriate perturbative cutoff. As briefly mentioned in Sect. 2, another option prompted by the geometry of our landscape might be to consider an alternate measure based on our angular Yukawa variable $\theta_{\lambda} \equiv \tan ^{-1} \lambda^{2}$ :

$$
d \theta_{\lambda}=\frac{d \lambda^{2}}{1+\lambda^{4}}
$$

Note that this measure suppresses the contributions from large $|\lambda|$, as we desired, yielding a finite result. For example, using this angular measure, we find that the average probability for finding stable extrema becomes

$$
\operatorname{Prob}(\text { stable })=\frac{2}{\pi} \int_{0}^{\pi / 2} d \theta_{\lambda} \operatorname{Prob}(\text { stable } ; \lambda)=\frac{151}{384} \approx 0.393 .
$$

One could also implement a perturbative cutoff for $\theta_{\lambda}$, again yielding cutoff-sensitive results. However, regardless of the particular measure or cutoffs chosen, this example illustrates in a graphic and concrete way the need to have an unambiguous way of determining appropriate measures and cutoffs in order to meaningfully calculate any statistical quantities on the landscape.

Another quantity that we can calculate in our toy model is the probability that a given (stable) vacuum state preserves $R$-symmetry. In our toy model, such $R$ symmetry-preserving vacua are those in Classes $\{\mathbf{1}\},\{2\}$, and $\{3\}$, while the vacua in Classes $\{\mathbf{1 2}\},\{\mathbf{1 3}\}$, and $\{\mathbf{2 3}\}$ have the potential to break $R$-symmetry. Thus, calculating the probability of randomly obtaining an $R$-symmetric vacuum from amongst all stable vacua as a function of $\lambda$, we obtain

$$
\operatorname{Prob}(\mathrm{R} \text { symm; } \lambda)= \begin{cases}(3 / \pi) \tan ^{-1} \lambda^{2} & \text { for } \lambda \leq 1 \\ (1 / 4)+(2 / \pi) \tan ^{-1} \lambda^{2} & \text { for } 1 \leq \lambda \leq \lambda_{*} \\ 1 & \text { for } \lambda \geq \lambda_{*}\end{cases}
$$

This probability is plotted in Fig. 8 as a function of $\lambda$.

\footnotetext{
${ }^{\ddagger}$ In a realistic setting, all three of these quantities may change dynamically under renormalizationgroup flow. This issue will be discussed further in Sect. 3.5. However, for the purposes of studying the vacuum structure of our toy model, we are considering these quantities as primordial fixed parameters defining our specific model.
} 


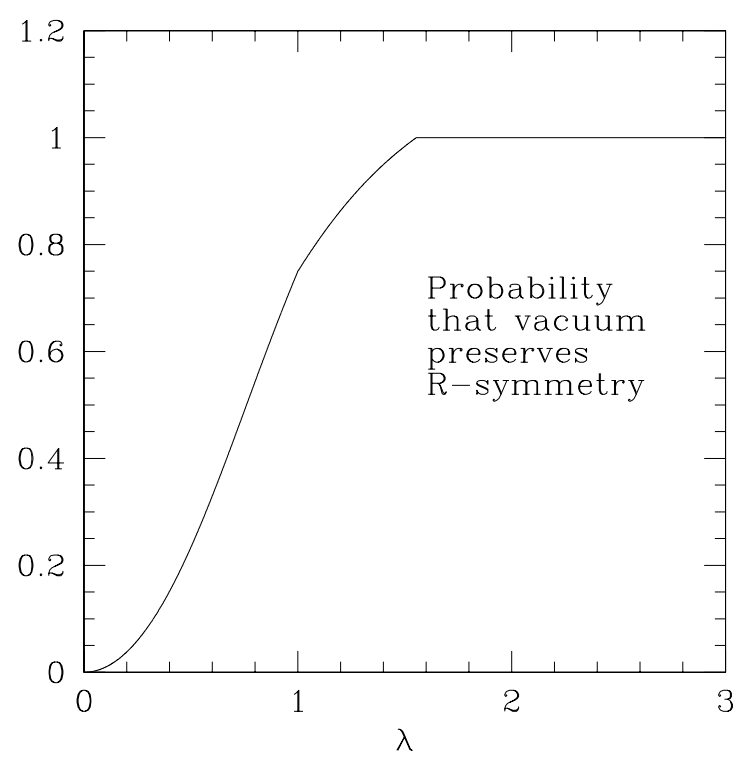

Figure 8: The probability that a given vacuum in our toy model preserves $R$-symmetry, plotted as a function of the Yukawa coupling $\lambda$ and integrated over all $\left(\xi_{1}, \xi_{2}\right)$. This function increases monotonically from zero when $\lambda=0$, and saturates at 1 for $\lambda \geq \lambda_{*}$.

Once again, just as with the probability of finding stable extrema, we recognize that we should really average this result over $\lambda$ (the third landscape coordinate). If we were to use the measure $\int_{0}^{\infty} d \lambda$ or $\int_{-\infty}^{\infty} d \lambda$, we would obtain the result that the average probability is one, since the contributions from large $|\lambda|$ (or from $|\lambda| \geq \lambda_{*}$ ) dominate over the contributions from smaller $\lambda$. However, using the "angular" measure in Eq. (3.13), we obtain the exact analytical result

$$
\operatorname{Prob}(\mathrm{R} \text { symm })=\frac{2}{\pi} \int_{0}^{\pi / 2} d \theta_{\lambda} \operatorname{Prob}(\mathrm{R} \text { symm } ; \lambda)=\frac{21}{32} \approx 0.656 .
$$

Clearly, this result depends strongly on the measure employed, and possible cutoffs for $\lambda$ that might be imposed.

\subsection{Renormalization-group flow and boundary crossings}

One of the interesting features of the vacuum structure we are seeing in this toy model is the fact that all of the boundaries between different vacuum regions are actually energy-dependent (or temperature-dependent in the early universe). Therefore, it is possible that a vacuum can cross a boundary between regions as the result of renormalization group evolution, either because the landscape location of the vacuum changes, because the boundary changes, or both.

In order to understand this potentially interesting observation, let us now analyze 
the evolution of our toy model under renormalization-group ( $R G)$ flow. As we have seen, this model actually contains several quantities which are potentially renormalized: these include the FI coefficients $\xi_{i}$ and the Yukawa coupling $\lambda$. However, we must remember that our toy model also depends on the $U(1)_{i}$ gauge couplings $g_{i}$ that were implicitly dropped from Eq. (3.2). In this model, the RGEs for the two gauge couplings $g_{1}, g_{2}$, and the Yukawa coupling $\lambda$ are given by

$$
\begin{aligned}
\mu \frac{d}{d \mu} g_{i} & =\frac{g_{i}^{3}}{16 \pi^{2}} \operatorname{Tr} Q_{i}^{2} \\
\mu \frac{d}{d \mu} \lambda & =\frac{\lambda}{16 \pi^{2}}\left(3 \lambda^{2}-4 g_{1}^{2}-4 g_{2}^{2}\right)
\end{aligned}
$$

where $\operatorname{Tr} Q_{i}^{2}$ is the sum of (squared) charges under $U(1)_{i}$.

In order to illustrate the phenomenon of boundary crossing, we will assume for simplicity that the entire matter content of our theory consists of the three chiral superfields $\Phi_{1}, \Phi_{2}$, and $\Phi_{3}$, and that the FI terms $\left(\xi_{1}, \xi_{2}\right)$ are introduced at tree level. We therefore find that $\operatorname{Tr} Q_{1}^{2}=\operatorname{Tr} Q_{2}^{2}=2$. Moreover, since $\operatorname{Tr} Q_{1}=\operatorname{Tr} Q_{2}=0$ in this case, the one-loop induced FI coefficients are zero, and therefore the tree-level FI coefficients $\left(\xi_{1}, \xi_{2}\right)$ are not renormalized. Thus, we see that in this toy model, the location of an individual vacuum in the landscape is invariant, and the only changes that can occur are those which change the topography of the landscape itself (moving boundaries, growing or shrinking regions, etc.) with respect to that fixed location.

Let us consider initial boundary values $g_{i}^{2}(\Lambda)=g_{i, 0}^{2}$ and $\lambda^{2}(\Lambda)=\lambda_{0}^{2}$, where $\Lambda$ is some initial reference ultraviolet (UV) scale. In order to make this calculation tractable, let us further assume that our gauge couplings are originally equal at the UV scale: $g_{1,0}=g_{2,0} \equiv g_{0}$. We therefore find from Eq. (3.17) that our gauge couplings remain equal at all subsequent energy scales: $g_{1}(\mu)=g_{2}(\mu) \equiv g(\mu)$.

The question that we wish to pursue, then, is that of determining the evolution of the entire landscape structure as a function of the energy scale $\mu$. We shall do this in several steps.

First, since $g_{1}(\mu)=g_{2}(\mu) \equiv g(\mu)$, we see that we can easily restore the gauge couplings to all of our previous landscape calculations simply by rescaling $\lambda(\mu) \rightarrow$ $\lambda(\mu) / g(\mu)$. This holds because the vacuum structure of the potential $V$ in Eq. (3.2) is not affected if we balance the rescaling of the $D$-terms with a corresponding rescaling of the $F$-terms. Of course, such a universal rescaling of $\lambda$ would not be possible if we did not assume that $g_{1,0}=g_{2,0}$.

Next, let us consider the sequence of landscape "snapshots" that we have already generated in Figs. 3, 4(a), 4(b), 5, 6(a), and 6(b). Reordering these figures, we see that they describe the landscape as a function of the parameter $\lambda(\mu)$, which should now be interpreted as the rescaled parameter $\lambda(\mu) / g(\mu)$. Therefore, if we wish to understand the $\mathrm{RG}$ flow in the landscape, we simply need to determine the flow of the single quantity $\lambda / g$ which parametrizes which landscape sketch is appropriate at which energy scale. Our original location on the landscape doesn't 
change with energy, but we simply have to look at the correct figure corresponding to the appropriate value of $\lambda / g$ in order to know the physics at this location. This observation is important, because it confirms that the $\mathrm{RG}$ flow simply corresponds to moving between landscape pictures while holding our original landscape location $\left(\xi_{1,0}, \xi_{2,0}\right)$ fixed. There is therefore no need to perform any RG calculation for the behavior of each individual boundary region.

Note that this last observation would not hold if we did not assume that $g_{1,0}=g_{2,0}$. In the case of unequal gauge couplings, our landscape pictures in previous sections might not apply, and new regions of stability/instability might emerge. However, because of the universal scaling argument which is possible when $g_{1,0}=g_{2,0}$, we see that our previous landscape calculations are guaranteed to apply regardless of $\mu$.

Thus, in order to determine the evolution between different landscape pictures, we simply need to determine the RG equation for our rescaled Yukawa coupling $Y(\mu) \equiv$ $\lambda(\mu) / g(\mu)$. For example, when $g_{1}(\mu)=g_{2}(\mu) \equiv g(\mu)$, the boundaries demarcating the $\{\mathbf{1}\}+\{\mathbf{3}\}$ overlap region for $Y(\mu)>1$ can be specified directly in terms of $Y(\mu)$ :

$$
-Y^{2}(\mu) \xi_{1}<\xi_{2}<-\frac{1}{Y^{2}(\mu)} \xi_{1}
$$

Given Eq. (3.17), it is relatively straightforward to determine the RG equation for $Y(\mu)$. We find

$$
\mu \frac{d}{d \mu} Y(\mu)=\frac{Y}{16 \pi^{2}}\left(3 Y^{2}-10\right) g^{2}(\mu)
$$

and substituting the explicit solution for $g^{2}(\mu)$ from Eq. (3.17), we can integrate Eq. (3.19) to obtain the solution

$$
3-\frac{10}{Y^{2}(\mu)}=\left(3-\frac{10}{Y_{0}^{2}}\right)\left[\frac{g(\mu)}{g_{0}}\right]^{10} .
$$

Thus, we see that quantity $3-10 / Y^{2}$ scales according to the ratio of gauge couplings $\left[g(\mu) / g_{0}\right]^{10}$. Since this ratio always decreases as we flow towards the infrared, we see that $3-10 / Y^{2}$ always evolves towards zero, either from above or below, as we flow towards the infrared. In other words, regardless of the initial value $Y_{0}$, our theory always flows towards an infrared fixed point

$$
\bar{Y} \equiv \sqrt{\frac{10}{3}} \approx 1.826
$$

The physical interpretation of this behavior is as follows. We begin our evolution with some initial values $\xi_{i, 0}$ (which determine our location in the landscape) as well as initial values $\lambda_{0}$ and $g_{0}$ (which determine which landscape sketch is appropriate). Our landscape location is invariant as we flow towards the infrared, but the underlying landscape picture (boundaries, etc.) will evolve. If we find that we are originally in 
a landscape with $Y_{0} \equiv \lambda_{0} / g_{0}<\bar{Y}$, we flow upwards through the landscape diagrams towards the landscape with $Y=\bar{Y}$. Conversely, if we are originally in a landscape with $Y_{0}>\bar{Y}$, we flow downwards through the landscape diagrams towards the landscape with $Y=\bar{Y}$. In all cases, however, the net result of the RG evolution towards the infrared is to wind up in the landscape corresponding to $Y=\bar{Y}$. This is the universal infrared limit.

Given this, it is straightforward to trace the RG dynamics that a single point in the landscape might experience. Note that a generic feature of the boundaries separating two different vacuum stability regions is the presence of a massless scalar in the spectrum. This is intuitively clear, since boundaries demarcate the stability regions of various vacua. Thus, crossing a boundary is in some sense equivalent to a phase transition, with the appearance of long-range order as the new massless state appears in the spectrum. The order of the phase transition can then be determined from the behavior of the vacuum energy $V$ as the boundary is crossed.

Note that in general, there are only two generic classes of boundaries that appear in our landscape diagrams for this toy model:

- Boundaries separating single-vev regions, such as $\{\mathbf{1}\}$, and two-vev regions, such as $\{\mathbf{1 2}\}$, in which one of the two vev's is the same as that in the singlevev region. These sorts of boundaries appear in Fig. 4(a), for example, and tend to dominate at small $Y$.

- Boundaries separating single-vev regions, such as $\{\mathbf{1}\}$, and overlap regions, such as $\{1\}+\{2\}$, in which one of the overlapping vacua is the same as the vacuum in the single-vev region. These sorts of boundaries appear in Fig 6(a), for example, and tend to dominate at large $Y$. Note that near the boundary, it is the common vacuum that has the lower vacuum energy $V$ in the overlap region, while the other vacuum in the overlap region is only metastable.

It is easy to verify that the vacuum energy $V$ is continuous across the first class of boundaries, while for the second class, the stable and the metastable vacua are not degenerate in energy on the boundary. Note that the only other types of boundaries are those that separate single-vev regions from each other (such as the boundary separating the $\{\mathbf{1}\}$ and $\{3\}$ regions in Fig. 3), but these are fine-tuned and occur only at the critical values $Y=1$ and $Y=Y_{*} \approx 1.55$ [where $Y_{*}$ corresponds to $\lambda_{*}$ in Eq. (3.11)].

Given these classes of boundaries, let us therefore consider the kinds of phase transitions which can result as a consequence of RG flow in our toy model.

If $Y_{0}>\bar{Y}$, then we find ourselves in a landscape containing only single-vev and overlap regions. Thus, we have only boundaries of the second type. Moreover, since $\mathrm{RG}$ flow pushes us towards landscapes with smaller values of $Y$, we can only have situations in which our overlap regions are getting smaller rather than larger. Thus, depending on our original (fixed) landscape location, the only type of boundary 
crossing that may occur in this case is one in which our location changes from being within an overlap region to within a single-vev region. For example, we may be originally be located in the $\{\mathbf{1}\}+\{\mathbf{3}\}$ overlap region, closer to the $\{\mathbf{1}\}$ region than to the $\{\mathbf{3}\}$ region. In such a case, as the boundary between the $\{\mathbf{1}\}+\{\mathbf{3}\}$ region and the $\{1\}$ region moves towards us and passes our location, we ultimately find ourselves in the $\{\mathbf{1}\}$ region.

In this case, the resulting physics depends on our initial vacuum state. Recall that in the overlap region, the true stable vacuum is determined according to the nearer single-vev region. For example, in $\{\mathbf{1}\}+\{\mathbf{3}\}$ overlap region, the $\{\mathbf{1}\}$ vacuum has lower energy than the (metastable) $\{\mathbf{3}\}$ vacuum if we are closer to the $\{\mathbf{1}\}$ region than to the $\{3\}$ region. Thus, if we are originally in the $\{\mathbf{1}\}$ state, then there is no effect as the boundary passes our location. However, if we are originally in the metastable $\{\mathbf{3}\}$ vacuum, this vacuum becomes unstable and the $\{\mathbf{1}\}$ solution becomes the only stable vacuum as the boundary passes our location. Of course, a key ingredient in making this determination is the timescale for boundary crossing compared with the timescale for decaying from the metastable $\{\mathbf{3}\}$ vacuum to the truly stable $\{\mathbf{1}\}$ vacuum even without a boundary crossing. If the latter timescale is shorter than the former, the decay from the metastable $\{3\}$ vacuum to the truly stable $\{\mathbf{1}\}$ vacuum can occur even before the boundary reaches us.

The analysis is slightly more complicated for $Y_{0}<\bar{Y}$. If $Y_{0}<1$, then our original landscape has only one-vev and two-vev regions, with the two-vev regions shrinking as a result of the RG flow towards larger $Y$-values. If we are originally located in a two-vev region of this landscape, then we will necessarily eventually experience a second-order phase transition into a one-vev vacuum as a result of $\mathrm{RG}$ flow. However, if $1<Y_{0}<Y_{*}$ [where $Y_{*}$ corresponds to $\lambda_{*}$ in Eq. (3.11)], then our landscape consists of a mixture of one-vev, two-vev, and overlap regions. As a result of RG flow towards greater $Y$-values, the two-vev regions are shrinking and the overlap regions are growing. Thus, two different types of transitions are possible: either we can be located in a two-vev region and experience a second-order phase transition into a one-vev region, as described above, or we can be originally located in a single-vev region next to a growing overlap region. In the latter case, our vacuum state in the single-vev region continues to be the truly stable vacuum state in the overlap region, so there is no phase transition. Finally, if $Y_{*}<Y_{0}<\bar{Y}$, we find ourselves in a landscape in which there are only single-vev regions and overlap regions, with the overlap regions growing as a result of RG flow. In such a case, as above, no phase transitions are possible: if we pass from a single-vev region into an overlap region, our original vacuum state continues to be the truly stable vacuum state in the overlap region, and no phase transition occurs.

Clearly, the possibility of such RG-induced phase transitions in the landscape is of great interest and relevance for cosmology, and in particular for inflationary models.

One important consequence of the infrared fixed-point behavior towards $Y=$ $\bar{Y} \approx 1.826$ follows directly from Eq. (3.15): our theory always flows in the infrared 
to one in which R-symmetry is preserved. This observation is true in our toy model regardless of the original landscape location or Yukawa/gauge couplings.

Indeed, the emergence of an infrared fixed point has an even more significant consequence: in such cases, the low-energy phenomenology becomes insensitive to the plethora of (ultimately string-theoretic) variables that define the ultraviolet landscape physics. If such infrared fixed points are generic features of the string-theoretic landscapes, their existence suggests that it may not be necessary to understand the full ultraviolet string theory in order to extract physically testable predictions from the landscape.

Of course, we have made a number of assumptions in performing our analysis in this section. In a more realistic situation, or with $g_{1,0} \neq g_{2,0}$, the true landscape topography is likely to be much more complicated, with an even richer set of possible phase transitions. Other important effects that we have ignored include, for example, the possibility of kinetic mixing [11]. Similarly, the RG evolution of the gauge couplings must clearly stop below the scale of gauge symmetry breaking; likewise, the beta-function coefficients depend on the scale of supersymmetry breaking in the sense that the matter spectrum is supersymmetric above this scale and non-supersymmetric below it. Such issues, while very important, are clearly model-dependent and must be studied case by case.

However, even in this simple toy model, we see that RG flow has great potential to lead to a number of novel phase transitions and boundary crossings in the landscape - effects which are highly sensitive to our initial landscape location $\left(\xi_{1,0}, \xi_{2,0}\right)$. Of course, as already emphased, we stress that the possible vacuum-structure phase transitions that we have discussed in this section must be understood in terms of temperature phase transitions in the early universe.

\subsection{Adding soft masses for the chiral fields}

Finally, before concluding this section, let us briefly comment on the effects which are induced in our toy model when non-zero masses are introduced for our chiral scalar fields. Towards this end, let us consider adding the mass term

$$
V_{\text {soft }}=\tilde{m}^{2} \sum_{i=1}^{3}\left|\phi_{i}\right|^{2}
$$

to the scalar potential given in Eq. (3.2).

It is straightforward to repeat the above vacuum-structure analysis for this case as well. Let us first focus on the case with $\lambda=0$. We then find that Class $\{\emptyset\}$ solutions, which were formerly unstable everywhere, now become stable in a small, central, triangular region given by

$$
\xi_{1}<\frac{1}{2} \tilde{m}^{2}, \quad \xi_{2}>-\frac{1}{2} \tilde{m}^{2}, \quad \xi_{2}-\xi_{1}<\frac{1}{2} \tilde{m}^{2} .
$$

Likewise, the solutions in Classes $\{\mathbf{1}\},\{\mathbf{2}\}$, and $\{\mathbf{3}\}$, which were also unstable everywhere for $\lambda=0$, now become stable within rectangular semi-infinite "strips" 
emanating outward from this central triangle. The final landscape is shown in Fig. 9. Note that as $\left(\xi_{1}, \xi_{2}\right) \rightarrow \pm \infty$ (i.e., as we move increasingly far from the origin or either axis), this diagram reduces back to that shown in Fig. 4(b). This is in accordance with our expectation that the effects of the mass terms should vanish if these masses are small compared with the Fayet-Iliopoulos (FI) coefficients. Of course, these masses remain important if either FI coefficient $\xi_{i}$ is small, or if their sum $\xi_{1}+\xi_{2}$ is small with $\xi_{1}<0, \xi_{2}>0$.

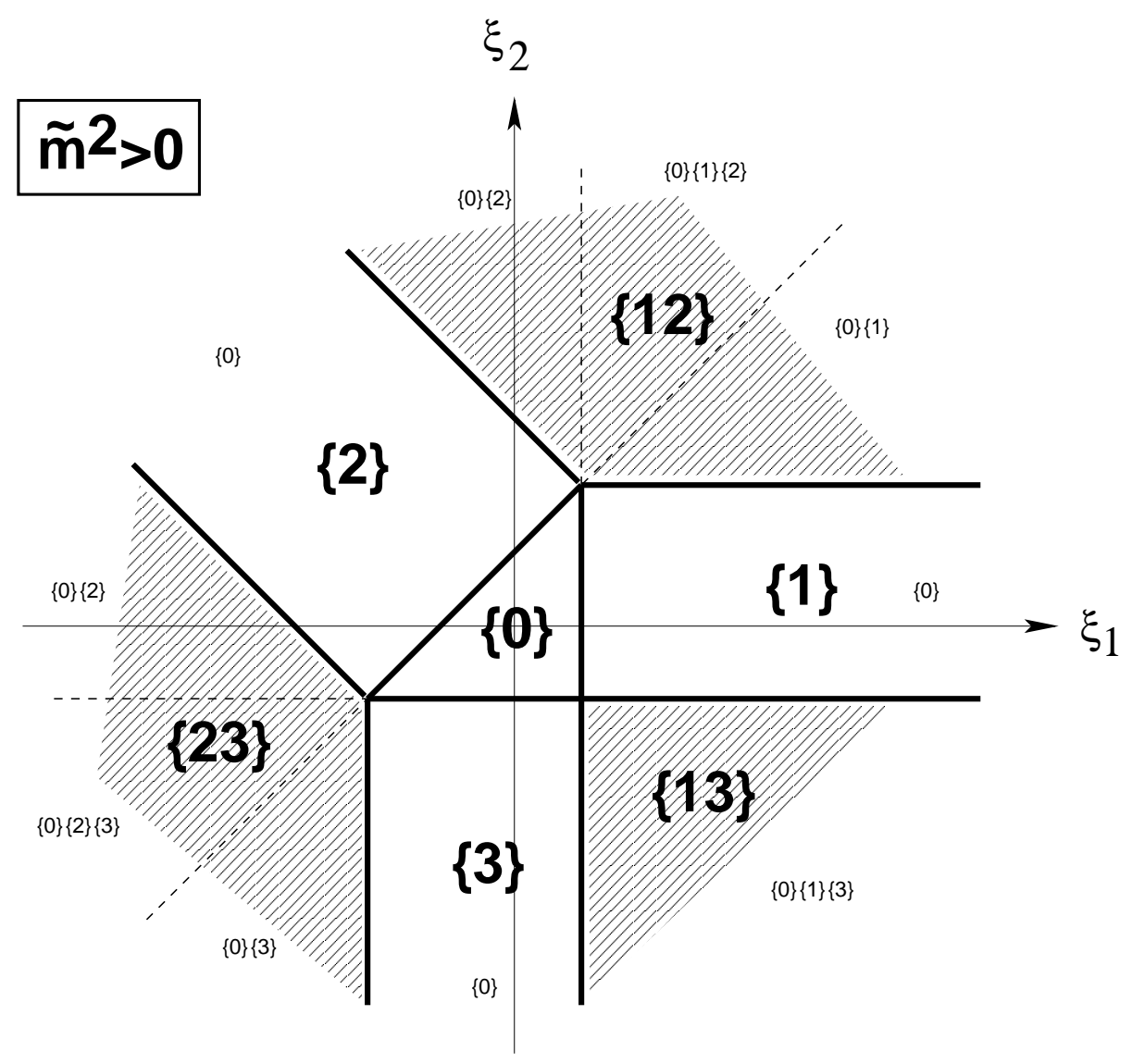

Figure 9: Same $\lambda=0$ landscape as shown in Fig. 4(b), but now with non-zero soft masses $\tilde{m}^{2}>0$ for the chiral superfields. The non-zero masses open up a central triangular region of stable vacua in Class $\{\emptyset\}$, and likewise introduce three semi-infinite rectangular "strips" of stable vacua in Classes $\{\mathbf{1}\},\{\mathbf{2}\}$, and $\{\mathbf{3}\}$. Note that very far from the origin, the relative effects of the mass terms generally vanish. In this limit, only those regions with non-zero opening angles survive, and the diagram reduces back to that shown in Fig. 4(b).

For $\lambda>0$, the situation generalizes as we might expect. First, we observe that stable $\{\emptyset\}$ vacua continue to exist within the triangular region in Eq. (3.23) for all $\lambda$, since this result is $\lambda$-independent. In general, for any $\lambda>0$, the one-vev stability regions then emanate outwards from the three sides of this central triangle, with the 
same opening angles as they have for $\tilde{m}^{2}=0$. Similarly, the stability regions that emanate from the vertices of this central triangle are the same as they would have been for $\tilde{m}^{2}=0$, corresponding either to two-vev vacua or to overlapping one-vev vacua depending on the value of $\lambda$. In all cases, we see that these landscapes with $\tilde{m}^{2}>0$ reduce back to the previous landscapes with $\tilde{m}^{2}=0$ when we look far from the origin and therefore retain only those stability regions with non-zero opening angles.

Finally, let us briefly consider the case with $\tilde{m}^{2}<0$. In this case, and with $\lambda=0$, it is easy to verify that no stable vacua exist anywhere in the landscape, i.e., for any values of $\left(\xi_{1}, \xi_{2}\right)$. This makes sense, since we already saw that the $\lambda=0$ landscape comprises a single flat direction when $\tilde{m}^{2}=0$, and this flat direction is necessarily destabilized for $\tilde{m}^{2}<0$. However, for non-zero $\lambda$, this expectation can change since we can balance the stabilizing $F$-terms against the destabilizing soft masses. In general, we find that as $\lambda$ increases from zero, stable pie-slice regions of stable vacua begin to exist at the outer edges of the landscape. These vacua have the same opening angles and properties as functions of $\lambda$ as they have in the $\tilde{m}^{2}=0$ case, but their origination points are displaced away from the origin, leaving a hollow, unstable center. Thus, this is our first example of a hole in the landscape - i.e., a bounded finite region of the landscape in which there are no stable vacua of any sort.

As $\lambda$ increases, each of these pie-slice regions begins to originate from a point closer and closer to the origin. Together, they eventually fill the hole completely when $\lambda$ reaches a critical value related to $|\tilde{m}|$. However, as $\lambda$ continues to increase beyond this critical value, each of these origination points continues to move past the origin, thereby creating a crowded new region of three overlapping single-vev vacua in the vicinity of the origin. This is thus our first example of more than two simultaneously overlapping vacua in the same landscape region.

\section{Adding more $\mathrm{U}(1)$ gauge groups}

In this section we shall consider generalizing the model in Sect. 3 by adding more $U(1)$ gauge groups. This will significantly increase the number of vacua and the complexity of the corresponding landscape. More importantly, since the Wilson-line superpotential can in principle contain more fields, we see from dimensional analysis that the $F$-terms will generically be suppressed. Thus, $R$-symmetry will be tend to be broken only at very low energies and only for relatively few vacua.

There are, of course, many different ways in which we can introduce further $U(1)$ gauge groups. The simplest example containing a large number of vacua is provided by considering $n$ copies of the Fayet-Iliopoulos model analyzed in Sect. 2. Such a setup is easily realized in string models containing large numbers of Abelian factors, corresponding to branes with significant geometric separations in the six-dimensional internal space. Such a model would clearly have $3^{n}$ classes of vacua, and requires the various Abelian gauge group factors to be anomalous in the string-theory sense. 
While this is certainly possible, one must bear in mind that in perturbative string constructions, the number of Abelian factors is limited by the total rank of the gauge group, as determined by Ramond-Ramond (RR) tadpole cancellation conditions. In $2^{n}$ of these classes of vacua, the Abelian gauge group is completely broken.*

\begin{tabular}{||c||ccccc||}
\hline \hline & $U(1)_{1}$ & $U(1)_{2}$ & $U(1)_{3}$ & $\ldots$ & $U(1)_{n}$ \\
\hline \hline$\Phi_{1}$ & -1 & & & & \\
$\Phi_{2}$ & 1 & -1 & & & \\
$\Phi_{3}$ & & 1 & -1 & & \\
$\vdots$ & & & $\ddots$ & $\ddots$ & \\
$\Phi_{n}$ & & & & 1 & -1 \\
$\Phi_{n+1}$ & & & & & 1 \\
\hline \hline
\end{tabular}

Table 2: $U(1)$ charge assignments for chiral superfields, inspired by "deconstruction" models of extra dimensions.

Things become less straightforward if the various Abelian factors are not decoupled from one another. For this purpose, let us therefore consider a generalization of the two- $U(1)$ model of Sect. 3 to the case of $n$ different $U(1)$ gauge group factors, with $n+1$ chiral superfields. Inspired by deconstruction models of extra dimensions [7], we shall take our charge assignments to follow the pattern indicated in Table 2. Thus, as evident from Table 2, we are only considering bi-fundamental "nearest-neighbor" charges; other configurations will be briefly discussed in Sect. 6. Likewise, we shall assume for simplicity that only the boundary $U(1)$ gauge-group factors, i.e., $U(1)_{1}$ and $U(1)_{n}$, have Fayet-Iliopoulos coefficients $\xi_{1}$ and $\xi_{n}$ respectively. Given these charges, we can in general write down a Wilson-line superpotential of the form ${ }^{\dagger}$

$$
W=\lambda \prod_{i=1}^{n+1} \Phi_{i}
$$

Note that unlike the previous case with $n=2$, this superpotential is not renormalizable for $n>2$. However, we can continue to study the landscape of this model as a function of the Fayet-Iliopoulos coefficients $\left(\xi_{1}, \xi_{n}\right)$ for arbitrary values of the Yukawa parameter $\lambda$. We can also consider the addition of soft scalar masses by adding a term

$$
V_{\text {soft }}=\tilde{m}^{2} \sum_{i=1}^{n+1}\left|\phi_{i}\right|^{2}
$$

to the scalar potential, as in Sect. 3.

* Models with several Abelian factors have been considered phenomenologically in various papers; see, e.g., Ref. [11] and references therein.

${ }^{\dagger}$ This implies that the $\Phi_{i}$ superfields have $R$-charge $2 /(n+1)$, giving the $F$-terms $R$-charge $-2 n /(n+1)$. Thus, as claimed earlier, $F$-term breaking will correspond to $R$-symmetry breaking in this model. 


\subsection{Special case $n=3$}

Let us first consider the case with $n=3$. Since the superpotential $W$ is nonrenormalizable, its contribution to the overall potential is negligible at energy scales below the fundamental physics scale. We shall therefore take $\lambda=0$ in our analysis. However, we shall continue to leave the common scalar soft mass $\tilde{m}^{2}$ arbitrary.

Since there are four chiral superfields when $n=3$, there are now sixteen possible classes of vacua which can be characterized by the vacuum expectation values (vev's) $v_{i}$ of the scalar fields $\phi_{i}$. We shall continue to employ a notation in which $\{\mathrm{ijk} . .$. denotes the class of extrema in which $v_{i}, v_{j}, v_{k}, \ldots$, are non-zero. Likewise, the notation $\{\emptyset\}$ will denote the class of extrema in which all vev's vanish. For example, $\{14\}$ denotes extrema in which $v_{2}=v_{3}=0$ while $v_{1} \neq 0, v_{4} \neq 0$. In general, just as in the $n=2$ case, we can calculate a $(8 \times 8)$-dimensional mass matrix as in Eq. (3.5). There will be a zero eigenvalue for each spontaneously broken $U(1)$; these eigenvalues correspond to the resulting Nambu-Goldstone bosons. Our extrema then represent stable (or metastable) vacua only if each of the remaining eigenvalues is positive.

We find the following results. In general, of the sixteen potential classes of extrema, we find that only eleven classes of extrema actually exist; there are no solutions for extrema in Classes $\{12\},\{34\},\{124\},\{134\}$, or $\{1234\}$.

Of the remaining eleven classes of extrema, we find that only three of these give rise to stable (non-overlapping) regions when $\tilde{m}=0$. This case is sketched in Fig. 10, which should be compared with the analogous $n=2$ case Fig. 4(b). Compared with the $n=2$ case, we see that the stable regions have a virtually identical shape, with the $\{\mathbf{1 2}\}$ vacua for $n=2$ corresponding to the $\{\mathbf{1 2 3}\}$ vacua for $n=3$; the $\{\mathbf{2 3}\}$ vacua for $n=2$ corresponding to the $\{234\}$ vacua for $n=3$; and the $\{13\}$ vacua for $n=2$ corresponding to the $\{\mathbf{1 4}\}$ vacua for $n=3$. There are some differences, however. For example, in the $n=2$ case, the entire landscape was dominated by vacua that break $R$-symmetry. By contrast, if we classify our $n=3$ vacua according to their general properties for arbitrary $\lambda$, we see that the $\{\mathbf{1 4}\}$ vacua are actually supersymmetric and consequently preserve $R$-symmetry. This is therefore our first example of a landscape containing both supersymmetric and non-supersymmetric regions of the same dimensionality [as opposed to lines of supersymmetric vacua within two- (or higher-)dimensional non-supersymmetric regions]. We also notice that the $n=3$ case gives rise to slightly more unstable extrema for each stable vacuum than we found in the $n=2$ case. Finally, we note that the dashed lines in Fig. 10 have slopes 2 and 1/2, as opposed to the single slope 1 in Fig. 4(b).

We stress that Fig. 10 must be interpreted in the same way as Fig. 4(b), namely as the landscape that emerges for infinitesimal $\lambda$ approaching zero. When $\lambda=0$, the entire landscape in Fig. 10 actually becomes supersymmetric and $R$-symmetry preserving, completely dominated by the flat directions implicit in the $\lambda=0$ solution $\{1234\}$.

We can also consider the effects of turning on the soft masses $\tilde{m}$. As expected 


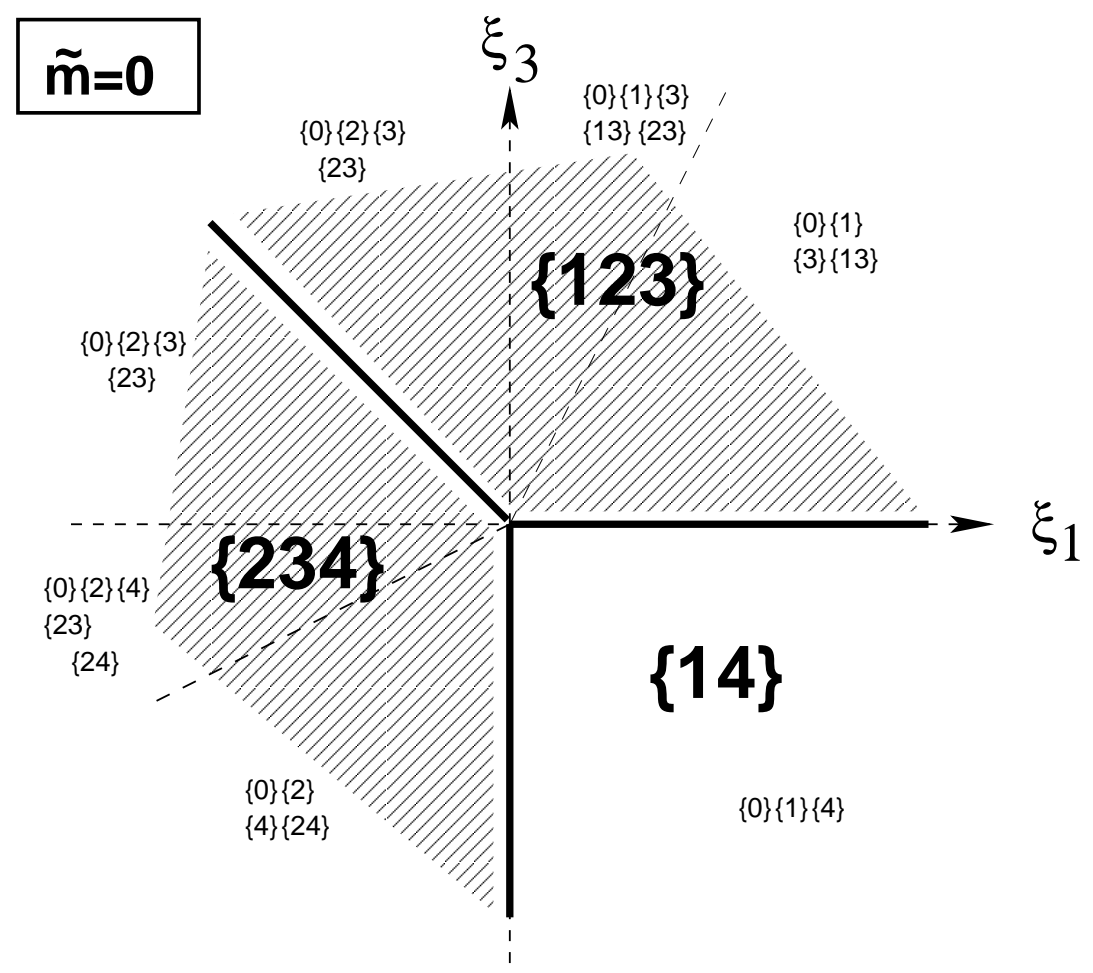

Figure 10: The landscape of our $n=3$ model for $\tilde{m}=0$. Stable vacua are indicated in large bold type, whereas unstable extrema are indicated in smaller non-bold type. Vacua breaking $R$-symmetry are shaded; note that the $\{\mathbf{1 4}\}$ region is supersymmetric and hence $R$-symmetry preserving. Compared with the analogous $n=2$ case in Fig. $4(\mathrm{~b})$, we see that not all vacua break $R$-symmetry for $n=3$. There are also slightly more unstable extrema for each stable vacuum.

from the $n=2$ case, we find that the $\{\emptyset\}$ solutions, which were formerly unstable everywhere, now become stable in a small, central, square region given by

$$
\left|\xi_{1}\right|<\tilde{m}^{2}, \quad\left|\xi_{3}\right|<\tilde{m}^{2}
$$

Likewise, of the seven remaining unstable classes of extrema, two of these (Classes $\{2\}$ and $\{3\}$ ) become stable within small triangular regions attached to this central square, while the remaining five classes of vacua now become stable within rectangular semi-infinite strips emanating outward from the central square and triangles. The resulting landscape is shown in Fig. 11. Detailed solutions for each class of extrema in this model are given in the Appendix. 


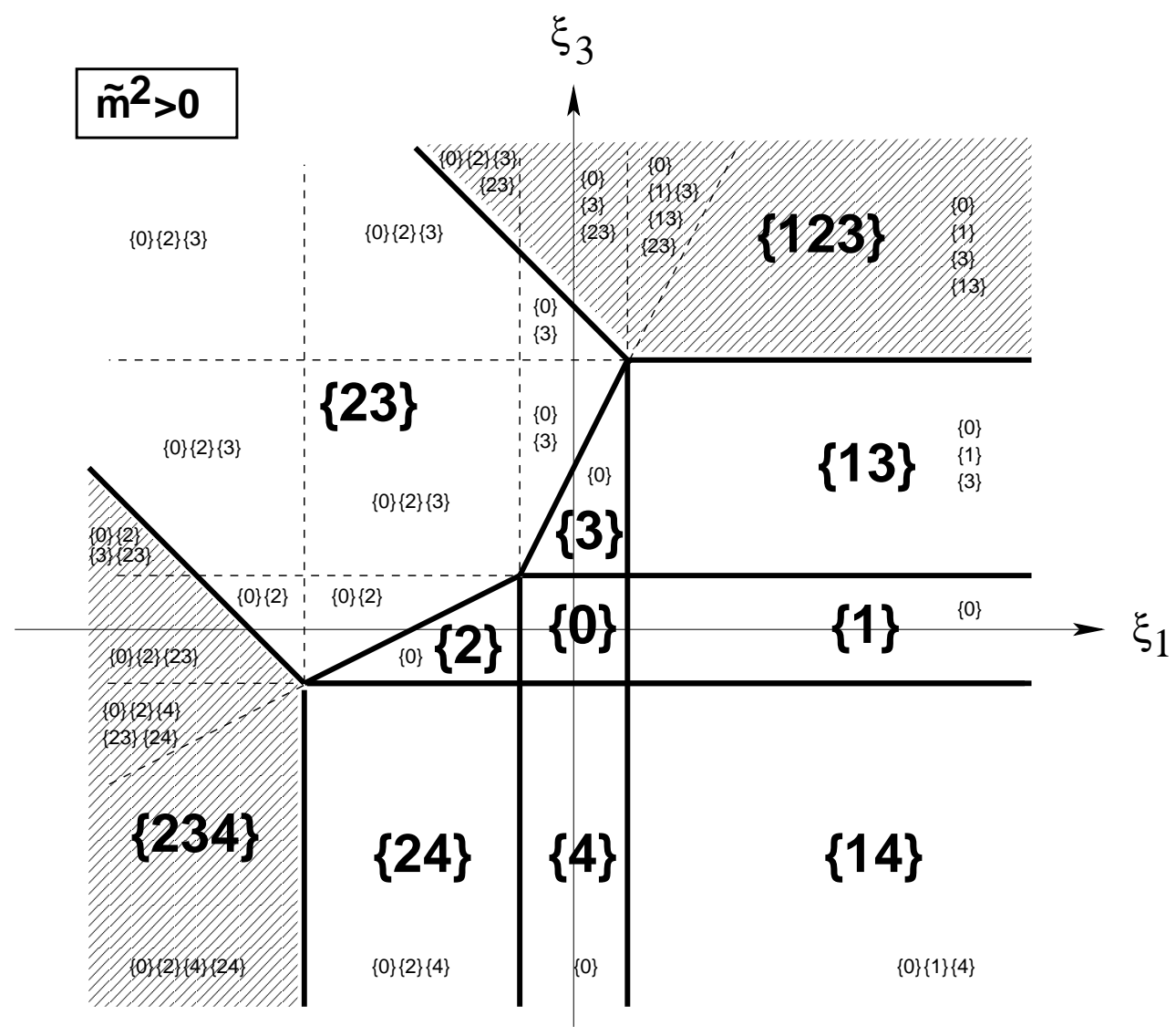

Figure 11: The landscape of our $n=3$ model, now drawn for $\tilde{m}^{2}>0$. Stable vacua are indicated in large bold type, whereas unstable extrema are indicated in smaller non-bold type. Vacua breaking $R$-symmetry are shaded. This landscape is to be compared with its $n=2$ equivalent in Fig. 9. Once again, we observe that in the $\tilde{m} \rightarrow 0$ limit (i.e., as we move radially outwards from the origin), only those regions with non-zero opening angles survive; this diagram then reduces back to that in Fig. 10.

\subsection{Arbitrary $n$ and large- $n$ limit}

We now turn our attention to the general- $n$ case, with particular interest in the large- $n$ limit. In the general case, the $D$ - and $F$-terms are now given by

$$
D_{a}=\sum_{i=1}^{n+1} q_{i}^{(a)}\left|\phi_{i}\right|^{2}+\xi_{1} \delta_{a 1}+\xi_{n} \delta_{a n}, \quad F_{i}=\frac{\partial W}{\partial \phi_{i}},
$$

where $a=1, \ldots, n$. This leads to the scalar potential

$$
V=\frac{1}{2} \sum_{a=1}^{n} D_{a}^{2}+\sum_{i=1}^{n+1}\left|F_{i}\right|^{2}
$$


As we already remarked for the $n=3$ case, the $F$-term contributions coming from the Wilson line superpotential (4.1) in the large- $n$ case have a negligible effect on the vacuum structure for FI terms smaller than the Planck (or string) scale. The extrema of the scalar potential are then obtained as solutions to the field equations

$$
\phi_{i}\left(q_{i}^{(1)} \xi_{1}+q_{i}^{(n)} \xi_{n}+\sum_{a=1}^{n} q_{i}^{(a)} \sum_{j=1}^{n+1} q_{j}^{(a)}\left|\phi_{j}\right|^{2}+|\lambda|^{2} \sum_{\substack{j=1 \\ j \neq i}}^{n+1}\left|\phi_{1} \ldots \hat{\phi}_{i} \ldots \hat{\phi}_{j} \ldots \phi_{n+1}\right|^{2}\right)=0
$$

where the hatted fields refer to fields that are omitted from the product. These equations each have two solutions: $v_{k} \equiv\left\langle\phi_{k}\right\rangle=0$, and a solution obtained by setting the term in parentheses to zero. This gives a large number $\sim \mathcal{O}\left(2^{n+1}\right)$ of different extrema which were explicitly analyzed in Sect. 3 for $n=2$ and Sect. 4.1 for $n=3$.

Certain features of the general- $n$ solution can easily be perceived directly by generalizing from the explicit $n=2$ and $n=3$ solutions. For example, it is straightforward to show that the $\tilde{m}=0, \lambda=0$ landscape always has three non-overlapping regions with the same shapes as in Fig. 6(b) and Fig. 10, and with the dashed lines (separating regions with different unstable extrema) having slopes $n-1$ and $1 /(n-1)$. Moreover, it is easy to see that the three regions of stability always correspond to vacua in the classes $\{1, \mathbf{n}+\mathbf{1}\},\{1,2,3, . ., \mathbf{n}\}$, and $\{2,3, \ldots, \mathbf{n}+\mathbf{1}\}$.

Similar general statements can also be made for the $\tilde{m} \neq 0$ case. When $\tilde{m}^{2}>0$, the presence of the non-zero soft mass always opens up a central region of stability for the $\{\emptyset\}$ extrema. In general, this region is an $(n+1)$-gon, with regions of stability for otherwise unstable extrema either emanating outwards in semi-infinite strips, or attached to the $(n+1)$-gon with bounded regions shaped like $n$-gons. Note that the appearance of a central $(n+1)$-gon can be understood as the result of having $n+1$ independent eigenvalue constraints defining the borders of the stability region.

While all of these properties hold true for general $n$, it is also instructive to examine certain specific vacuum solutions. Instead of attempting a general classification, we will concentrate on presenting some general features such as the absolute minima of the theory.

In the following, we shall focus on the case in which $\lambda / M_{P}^{n-2} \rightarrow 0$ and $\tilde{m}=0$. Since $\lambda / M_{P}^{n-2} \rightarrow 0$, we expect that the stable vacua are dominated by the three classes $\{\mathbf{1}, \mathbf{n}+\mathbf{1}\},\{\mathbf{1}, \ldots, \mathbf{n}\}$, and $\{\mathbf{2}, \ldots, \mathbf{n}+\mathbf{1}\}$. Specifically, we find that the $\{\mathbf{1}, \mathbf{n}+\mathbf{1}\}$ solution exists for $\xi_{1}>0, \xi_{n}<0$, and is given by

$$
\{\mathbf{1}, \mathbf{n}+\mathbf{1}\}: \quad\left|v_{1}\right|^{2}=\xi_{1}, \quad\left|v_{n+1}\right|^{2}=-\xi_{n}, \quad v_{2}=\ldots=v_{n}=0 .
$$

Similarly, the $\{\mathbf{1}, \ldots, \mathbf{n}\}$ solution exists for $\xi_{n}>0, \xi_{1}+\xi_{n}>0$, and is given by

$$
\begin{aligned}
\{\mathbf{1}, \ldots, \mathbf{n}\}: \quad & \left|v_{1}\right|^{2}=\left(\xi_{1}+\xi_{n}\right)\left(1+\mathcal{O}\left(\epsilon_{n}^{2}\right)\right) \\
& \left|v_{2}\right|^{2}, \ldots,\left|v_{n}\right|^{2}=\xi_{n}\left(1+\mathcal{O}\left(\epsilon_{n}^{2}\right)\right), \quad v_{n+1}=0
\end{aligned}
$$


where $\epsilon_{n}^{2} \sim\left(\lambda / M_{P}^{n-2}\right)^{2}\left(\xi_{n} / M_{P}^{2}\right)^{n-2}$, while the $\{\mathbf{2}, \ldots, \mathbf{n}+\mathbf{1}\}$ solution exists for $\xi_{1}<0$, $\xi_{1}+\xi_{n}<0$, and is given by

$$
\begin{aligned}
\{\mathbf{2}, \ldots, \mathbf{n}+\mathbf{1}\}: \quad & v_{1}=0, \quad\left|v_{n+1}\right|^{2}=-\left(\xi_{1}+\xi_{n}\right)\left(1+\mathcal{O}\left(\epsilon_{1}^{2}\right)\right) \\
& \left|v_{2}\right|^{2}, \ldots,\left|v_{n}\right|^{2}=-\xi_{1}\left(1+\mathcal{O}\left(\epsilon_{1}^{2}\right)\right)
\end{aligned}
$$

where $\epsilon_{1}^{2} \sim\left(\lambda / M_{P}^{n-2}\right)^{2}\left(\xi_{1} / M_{P}^{2}\right)^{n-2}$. Note that $\epsilon_{1}$ and $\epsilon_{n}$ are very small numbers for $n \gg 1$ and $\xi_{i} \ll M_{P}^{2}$. The vacuum in Eq. (4.8) was discussed in detail in Ref. [12]. Each of these classes of vacua occupy non-overlapping regions in the twodimensional $\left(\xi, \xi_{n}\right)$ parameter space. Note that the $\{\mathbf{1}, \mathbf{2}, \ldots, \mathbf{n}\}$ and $\{\mathbf{2}, \mathbf{3}, \ldots, \mathbf{n}+\mathbf{1}\}$ vacua completely break all of the $U(1)$ gauge factors, while the $\{\mathbf{1}, \mathbf{n}+\mathbf{1}\}$ vacuum is supersymmetric and $\lambda$-independent for all $n \geq 3$.

We shall also be interested in several other explicit solutions for general $n$. All of the following solutions are $\lambda$-independent. For example, the $\{\mathbf{2}, \mathbf{3}, \ldots, \mathbf{n}\}$ solution is given by

$$
\begin{array}{ll}
\{\mathbf{2}, \mathbf{3}, \ldots, \mathbf{n}\}: \quad & v_{1}=v_{n+1}=0, \\
& \left|v_{k}\right|^{2}=\frac{1}{n}\left[\left(\xi_{1}+\xi_{n}\right)(k-1)-n \xi_{1}\right] \text { for } k=2, \ldots, n .
\end{array}
$$

This solution has an unbroken $U(1)$ generator $Q_{1}+\ldots+Q_{n}$, where $Q_{i}$ are the generators of $U(1)_{i}$, and gives rise to the $D$-terms

$$
\left\langle D_{1}\right\rangle=\ldots=\left\langle D_{n}\right\rangle=\frac{\xi_{1}+\xi_{n}}{n}
$$

Note that this solution has a linear "profile", in the sense that the sequence of nonzero vacuum expectation values $\left|v_{k}\right|^{2}$ in Eq. (4.10) grows linearly with $k$.

Another solution of interest is the $\{\mathbf{2}, \mathbf{3}, \ldots, \mathbf{n}-\mathbf{1}, \mathbf{n}+\mathbf{1}\}$ solution, given by

$$
\begin{array}{ll}
\{\mathbf{2}, \mathbf{3}, \ldots, \mathbf{n}-\mathbf{1}, \mathbf{n}+\mathbf{1}\}: \quad & v_{1}=v_{n}=0, \quad\left|v_{n+1}\right|^{2}=-\xi_{n} \\
& \left|v_{k}\right|^{2}=\frac{k-n}{n-1} \xi_{1} \quad \text { for } k=2, \ldots, n-1 .
\end{array}
$$

This solution has the unbroken generator $Q_{1}+Q_{2}+\cdots+Q_{n-1}$, and gives rise to the $D$-terms

$$
\left\langle D_{1}\right\rangle=\ldots=\left\langle D_{n-1}\right\rangle=\frac{\xi_{1}}{n-1}, \quad\left\langle D_{n}\right\rangle=0
$$

This solution also exhibits a linear profile.

Another solution of interest is the $\{\mathbf{1}, \mathbf{3}, \mathbf{4}, \ldots, \mathbf{n}\}$ solution, given by

$$
\begin{array}{lll}
\{\mathbf{1}, \mathbf{3}, \mathbf{4}, \ldots, \mathbf{n}\}: \quad & v_{2}=v_{n+1}=0, \quad\left|v_{1}\right|^{2}=\xi_{1} \\
& \left|v_{k}\right|^{2}=\frac{k-2}{n-1} \xi_{n} & \text { for } k=3,4, \ldots, n .
\end{array}
$$


This solution has an unbroken $U(1)$ generator $Q_{2}+\ldots+Q_{n}$, and gives rise to the $D$-terms

$$
\left\langle D_{1}\right\rangle=0,\left\langle D_{2}\right\rangle=\cdots=\left\langle D_{n}\right\rangle=\frac{\xi_{n}}{n-1} .
$$

Clearly, this solution also exhibits a linear profile.

Finally, the last solutions we shall consider are the $\{\mathbf{2}, \mathbf{3}, \ldots, \mathbf{j}-\mathbf{1}, \mathbf{j}+\mathbf{1}, \ldots, \mathbf{n}\}$ solutions, given by

$$
\begin{aligned}
& \{\mathbf{2}, \mathbf{3}, \ldots, \mathbf{j}-\mathbf{1}, \mathbf{j}+\mathbf{1}, \ldots, \mathbf{n}\}: \quad v_{1}=v_{j}=v_{n+1}=0 \\
& \left|v_{k}\right|^{2}= \begin{cases}\frac{k-j}{j-1} \xi_{1} & \text { for } k=2, \ldots, j-1 \\
\frac{k-j}{n-j+1} \xi_{n} & \text { for } k=j+1, \ldots, n .\end{cases}
\end{aligned}
$$

These solutions leave unbroken the two generators $Q_{1}+Q_{2}+\ldots+Q_{j-1}$ and $Q_{j}+$ $Q_{j+1}+\ldots+Q_{n}$, and gives rise to the $D$-terms

$$
\begin{aligned}
& \left\langle D_{1}\right\rangle=\cdots=\left\langle D_{j-1}\right\rangle=\xi_{1} /(j-1) \equiv d_{1} \\
& \left\langle D_{j}\right\rangle=\cdots=\left\langle D_{n}\right\rangle=\xi_{n} /(n-j+1) \equiv d_{2} .
\end{aligned}
$$

Unlike the previous solutions, each of these solutions has two independent linear profiles.

Needless to say, there are numerous other solutions which can be generated for general $n$. However, the above solutions will be sufficient for our purposes.

Note that these vacua, as well as all vacua with smaller numbers of non-zero vev's,

are unstable at the level of our discussion (in which we are taking $\lambda / M_{P}^{n-2} \rightarrow 0$ and $\tilde{m}=0$ ). Moreover, one of the obvious properties of such solutions is the fact that, at first sight, they unfortunately appear to give rise to a large number of unbroken Abelian gauge symmetries which would survive down to low energies. However, as we shall see in Sect. 4.4, this is not the case due to the presence of mixed gauge anomalies.

\subsection{Higher-dimensional flux interpretations}

We shall now demonstrate that many of the above general- $n$ solutions have natural interpretations in terms of higher-dimensional flux compactifications.

First, let us consider the $\{\mathbf{1}, \mathbf{2}, \ldots, \mathbf{n}\}$ and $\{\mathbf{2 , 3}, \ldots, \mathbf{n}+\mathbf{1}\}$ vacua. These clearly can be interpreted as emerging from a five-dimensional supersymmetric $U(1)$ gauge theory compactified on the orbifold $S^{1} / \mathbb{Z}_{2}$, with compactification radii

$$
R \sim n / \sqrt{\xi_{n}} \text { and } \sim n / \sqrt{\left|\xi_{1}\right|}
$$

respectively. In each case, the four-dimensional zero-mode gauge field receives a mass from the four-dimensional FI term $\xi_{1}+\xi_{n}$. The supersymmetry-breaking scale 
in these two cases is controlled by the Wilson-line superpotential, and all soft masses are $\sim \mathcal{O}\left(\epsilon_{n}\right)$ in the first case and $\sim \mathcal{O}\left(\epsilon_{1}\right)$ in the second case. By contrast, the third vacuum $\{\mathbf{1}, \mathbf{n}+\mathbf{1}\}$ is supersymmetric and probably does not have an extradimensional interpretation.

We now turn to the $\{\mathbf{2}, \mathbf{3}, \ldots, \mathbf{n}\}$ solution in Eq. (4.10). We shall now argue that this vacuum can be given the higher-dimensional interpretation of having magnetic flux on a torus in a six-dimensional Abelian gauge theory. Indeed, as we will see, the smoking gun for such a magnetic flux interpretation is the presence of a linear profile in the associated vacuum expectation values.

From a five-dimensional $\mathbb{R}^{4} \times S^{1} / \mathbb{Z}_{2}$ perspective, where the fifth dimension is the interval $0<y<\pi R$, a supersymmetric Abelian gauge theory contains a gauge field and a $\mathbb{Z}_{2}$-odd real scalar $\Sigma$. The $D$-term from the four-dimensional point of view in the continuous limit of the deconstruction setup discussed above is given by

$$
D=\partial_{5} \Sigma+\left(-\left|\phi_{1}\right|^{2}+\xi_{1}\right) \delta(y)+\left(\left|\phi_{n+1}\right|^{2}+\xi_{n}\right) \delta(y-\pi R) \text {. }
$$

The standard profile for the scalar $\Sigma$, largely discussed in the literature, is of the form $\langle\Sigma\rangle=\epsilon(y) \xi_{n} / 2$, which in the case $\xi_{1}+\xi_{n}=0$ is the needed profile for preserving supersymmetry and the gauge symmetry [13]. Notice, however, that the field equations

$$
\delta \Sigma\left\{\partial_{4}^{2} \Sigma+\partial_{5}\left[\partial_{5} \Sigma+\left(\xi_{1}-\left|v_{1}\right|^{2}\right) \delta(y)+\left(\xi_{n}+\left|v_{n+1}\right|^{2}\right) \delta(y-\pi R)\right]\right\}=0
$$

have another solution on the orbifold $S_{1} / \mathbb{Z}_{2}$, namely

$$
\langle\Sigma\rangle=\frac{\xi_{1}+\xi_{n}}{2 \pi R} y-\frac{\xi_{1}}{2} \epsilon(y), \quad\langle D\rangle=\frac{\xi_{1}+\xi_{n}}{2 \pi R}
$$

This solution does not describe the absolute minimum of the theory, since it has a large positive vacuum energy, but it is an extremum of the theory. By using $R \sim n$, it is clear that Eq. (4.21) matches the deconstructed result Eq. (4.10). On the other hand, from a six-dimensional perspective, $\Sigma$ corresponds to the sixth-component $A_{6}$ of the gauge field. Then the flux in the two-dimensional compact space is given by

$$
F_{56} \equiv\left\langle\partial_{5} \Sigma\right\rangle=\frac{\xi_{1}+\xi_{n}}{2 \pi R}-\xi_{1} \delta(y)-\xi_{n} \delta(y-\pi R)
$$

The first term is the magnetic flux we were searching for, whereas the localized terms, already discussed in the literature, have the interpretation of fluxes localized at the orbifold fixed points. Note that the integrated flux in the compact space is actually zero,

$$
\int_{-\pi R}^{\pi R} d y\left\langle\partial_{5} \Sigma\right\rangle=0
$$

the magnetic flux cancelling the localized contributions at the fixed points. 
The other extrema listed in Sect. 4.2 also have a flux-compactification interpretation in extra dimensions. For example, let us consider the $\{\mathbf{2}, \mathbf{3}, \ldots, \mathbf{n}-\mathbf{1}, \mathbf{n}+\mathbf{1}\}$ solution given in Eq. (4.12). In the extra-dimensional interpretation, the bulk field $\Sigma$ has a different profile:

$$
\langle\Sigma\rangle=\frac{\xi_{1}}{2 \pi R} y-\frac{\xi_{1}}{2} \epsilon(y), \quad\langle D\rangle= \begin{cases}\xi_{1} /(2 \pi R) & \text { for } 0 \leq y<\pi R \\ 0 & \text { for } y=\pi R\end{cases}
$$

This gives rise to a flux which again integrates to zero:

$$
F_{56} \equiv\left\langle\partial_{5} \Sigma\right\rangle=\frac{\xi_{1}}{2 \pi R}-\xi_{1} \delta(y), \quad \int_{-\pi R}^{\pi R} d y\left\langle\partial_{5} \Sigma\right\rangle=0 .
$$

It may seem strange that the discontinuity at $y=\pi R$ in the auxiliary field $D$ can be consistent with the field equations (4.20). However, in Eq. (4.24) we have $\langle\Sigma(\pi R)\rangle=0$, and by choosing the consistent boundary condition $\delta \Sigma(\pi R)=0$, the field equations (4.20) are indeed satisfied.

This will be the general rule for the flux interpretation of the solutions with lower numbers of non-zero vev's: discontinuities in the auxiliary $D$-terms (and therefore the flux) correspond to points where the wave function of $\Sigma$ has Dirichlet boundary conditions. A solution with $\ell$ zero vev's for the link fields therefore corresponds to a wave function of $\Sigma$ having $\ell$ nodes (and corresponding Dirichlet boundary conditions) on the corresponding points. The solution in Eq. (4.12) is also unstable, having a tachyon at the position $\left(\phi_{n}\right)$ of the link field having a zero vev, but by adding the Wilson-line superpotential contributions, this solution becomes a stable vacuum close to the vertical axis $\xi_{1}=0$.

The final example with $n-1$ non-zero vev's is the $\{\mathbf{1}, \mathbf{3}, \mathbf{4}, \ldots, \mathbf{n}\}$ solution given in Eq. (4.14). In the extra-dimensional interpretation, the bulk field $\Sigma$ has the profile

$$
\langle\Sigma\rangle=\frac{\xi_{n}}{2 \pi R} y, \quad\langle D\rangle= \begin{cases}\xi_{n} /(2 \pi R) & \text { for } 0<y \leq \pi R \\ 0 & \text { for } y=0\end{cases}
$$

leading to a flux

$$
F_{56}=\frac{\xi_{n}}{2 \pi R}-\xi_{n} \delta(y-\pi R), \quad \int_{-\pi R}^{\pi R} d y\left\langle\partial_{5} \Sigma\right\rangle=0 .
$$

Moreover, consistent with our previous explanations, $\langle\Sigma\rangle$ vanishes at the position $y=0$ where the auxiliary field is discontinuous. Just as with the two previous flux solutions, this solution is unstable. However, by adding the Wilson-line superpotential, this solution becomes stable close to the horizontal axis $\xi_{n}=0$.

The last examples we shall discuss are the $\{\mathbf{2}, \mathbf{3}, \ldots, \mathbf{j}-\mathbf{1}, \mathbf{j}+\mathbf{1}, \ldots, \mathbf{n}\}$ solutions. In these cases, the profile for the bulk scalar $\Sigma$ satisfies

$$
\begin{aligned}
\delta \Sigma\left\{\left\langle\partial_{5} \Sigma\right\rangle+\right. & \left.\xi_{1} \delta(y)+\xi_{n} \delta(y-\pi R)\right\} \\
& =\delta \Sigma\left\{d_{2}+\frac{1}{2}\left(d_{2}-d_{1}\right)\left[\epsilon\left(y-y_{j}\right)-\epsilon\left(y+y_{j}\right)\right]\right\}
\end{aligned}
$$


where $y_{j}$ is the location at which the auxiliary field $\langle D\rangle$ jumps from $\langle D\rangle=d_{1}$ for $0<|y|<y_{j}$ to $\langle D\rangle=d_{2}$ for $y_{j}<|y|<\pi R$. The profile has the form

$$
\langle\Sigma\rangle= \begin{cases}d_{1} y-\frac{\xi_{1}}{2} \epsilon(y) & \text { for } 0<|y|<y_{j} \\ d_{2} y+\left(\frac{1}{2} \xi_{n}-\pi R d_{2}\right) \epsilon(y) & \text { for } y_{j}<|y|<\pi R\end{cases}
$$

where the constraint

$$
d_{1} y_{j}+d_{2}\left(\pi R-y_{j}\right)=\frac{1}{2}\left(\xi_{1}+\xi_{n}\right)
$$

serves as the continuity condition for the profile at $y=y_{j}$. By matching the deconstructed version to the continuous version via $y_{j} \sim j-1$, we find the expected result that $\left\langle\Sigma\left(y_{j}\right)\right\rangle=0$. The flux in this case is

$$
F_{56}=d_{2}+\frac{1}{2}\left(d_{2}-d_{1}\right)\left[\epsilon\left(y-y_{j}\right)-\epsilon\left(y+y_{j}\right)\right]-\xi_{1} \delta(y)-\xi_{n} \delta(y-\pi R),
$$

which again integrates to zero.

\subsection{Anomalies in the deconstructed $U(1)^{n}$ theory}

As we remarked earlier, the solutions with $n-1$ non-zero vev's - and indeed all of the solutions with fewer non-zero vev's - are unstable at the level of our discussion (in which we are taking $\lambda / M_{P}^{n-2} \rightarrow 0$ and $\tilde{m}=0$ ). However, at first sight it may also seem that these vacua have a further unpleasant feature, namely a large number of unbroken Abelian gauge symmetries which survive down to low energies. However, as we shall now demonstrate, this is not the case thanks to the presence of mixed gauge anomalies in such theories.

It is easy to see why subtleties related to mixed anomalies will be present in any model containing multiple Abelian gauge fields related to higher-dimensional compactifications and/or string theory. The basic reason is that bi-fundamental fields, which have been the building blocks of our toy landscape models, create mixed anomalies which typically require axionic fields and four-dimensional Green-Schwarz mechanisms for their cancellation. The axionic fields will come in complex (moduli) superfields due to supersymmetry, which will also fix the values of the FI terms of the model. The dynamics and the stabilization of these moduli is, of course, very important in the landscape picture. Indeed, we have thus far been assuming that these moduli are already stabilized by the dynamics (through, e.g., NS-NS and RR fluxes, gaugino condensation, etc.).

In the $U(1)^{n}$ example, the mixed anomalies are described by the $(n \times n)$ dimensional anomaly matrix

$$
C_{a b}=\frac{1}{4 \pi^{2}} \operatorname{Tr}\left(Q_{a} Q_{b}^{2}\right) \quad(a, b=1, \ldots, n),
$$


where $Q_{a}$ is the generator of the $U(1)_{a}$ gauge group. The explicit matrix elements of $C_{a b}$ are given by [12]

$$
C_{a b}=\frac{1}{4 \pi^{2}}\left[\delta_{a, b-1}-\delta_{a, b+1}+\operatorname{Tr} Q_{1}^{3} \delta_{a, 1} \delta_{b, 1}+\operatorname{Tr} Q_{n}^{3} \delta_{a, n} \delta_{b, n}\right]
$$

where for generality we have allowed for additional particles (e.g., MSSM particles) at the fixed points. Otherwise, in our minimal example discussed earlier, we would have $\operatorname{Tr} Q_{1}^{3}=\operatorname{Tr} Q_{n}^{3}=0$. Whereas at the four-dimensional level the anomaly-cancelling mechanism is not uniquely fixed (and we shall shortly provide a trivial illustrative example of this), the requirement of realizing a five-dimensional Lagrangian in the continuous limit is much more constraining. This was consistently done in Ref. [12], where it was shown that the axionic-type fields taking care of the mixed anomalies in Eq. (4.33) are themselves bi-fundamental. More precisely, there are axionic couplings of the type $-\ln \left(\phi_{i} \phi_{j}\right) \delta_{i a} \delta_{j, a+1} W_{a}^{\alpha} W_{\alpha, a}$, in superfield notation. There are also two boundary axionic fields $S_{L, R}$ which transform only under the first and the last gauge group. $\ddagger$

The presence of the non-minimal kinetic (and Kähler) functions clearly modifies the minimization procedure. It is therefore important to verify that our previous results remain intact. We have explicitly verified that the absolute minima of the model, as well as the first $(n-1)$-vev example with a clean magnetic flux interpretation, are affected in the full theory only at subleading order in $\xi_{i} / M_{P}^{2}$. However, the vacua with fewer than $(n-1)$ non-zero vev's do not remain extrema in the full theory, since a vanishing vev for a bi-fundamental represents a singular point of the theory. The natural solution in the case where a zero vev occurs in a link field $\phi_{k}$ is, in the extra-dimensional interpretation, to add a brane at the corresponding point in the compact space. We should also introduce a superfield $S_{k}$ with the gauge transformation $\delta S_{k} \sim \Lambda_{k-1}-\Lambda_{k}$, and replace $\ln \phi_{k} \rightarrow S_{k}$ in the gauge kinetic functions. This new field would then have the interpretation of coupling to the $U(1)$ gauge field of the brane. This automatically cancels the anomalies, explains why a new massless gauge field appears in the spectrum, and gives a rationale for the Dirichlet boundary condition which we found in Sect. 4.3 at the position of the vanishing vev.

This new axionic coupling can, however, render the new gauge field massive. Indeed, more and more vanishing vev's require the addition of more and more $U(1)$ branes and corresponding new axionic couplings, which eventually render most of the new brane $U(1)$ 's massive.

If we completely abandon the five-dimensional interpretation, there is certainly more freedom in anomaly cancellation. For example, we can introduce $n$ moduli fields $S_{a}$ which have $U(1)_{a}$ gauge transformations $\delta S_{1} \sim-\Lambda_{2}, \delta S_{a} \sim \Lambda_{a-1}-\Lambda_{a+1}$ for $a=2, \ldots, n-1$, and $\delta S_{n} \sim \Lambda_{n-1}$ where $\Lambda_{a}$ is the superfield describing $U(1)_{a}$ gauge transformations. Coupling these moduli fields to the gauge fields through a term of the form $\sum_{a} S_{a} W_{a}^{\alpha} W_{\alpha, a}$ will indeed cancel the mixed anomalies. In this case,

\footnotetext{
${ }^{\ddagger}$ Further details and a discussion of the five-dimensional limit are given in Ref. [12].
} 
assuming an a priori stabilization of moduli, all the results of our toy model follow accordingly. Generically however, this generates FI terms at all sites (i.e., for all $U(1)$ 's), unless the Lagrangian of the moduli fields and their vev's are such that FI terms vanish at all sites but the first and last. Indeed, such examples are relatively easy to find. However, the price to pay in such a construction is the loss of a fivedimensional interpretation in the limit of large $n$, and the unsolved remaining issue of moduli stabilization fixing the FI terms.

The upshot of the present discussion, then, is that in order to cancel gauge anomalies, we must introduce new axionic (super)fields with appropriate couplings under the various gauge groups. In this case, most of the $U(1)$ gauge-group factors become massive, a welcome feature for the phenomenological viability of these models.

\section{$5 \quad$ Extension to supergravity}

Among the main motivations for seriously considering the landscape picture of string theory are its ramifications for vacuum stability and the cosmological constant problem. From the perspective of both of these problems, the inclusion of supergravity interactions is of crucial importance. We shall now briefly discuss each of these issues in turn.

\subsection{Vacuum stability, soft masses, and split supersymmetry}

If we consider only the Abelian gauge fields which are relevant for our purposes, the scalar supergravity potential is given in terms of the Kähler potential $K$ and the superpotential $W$ by [14]

$$
\begin{aligned}
V\left(\phi_{i}\right)=e^{K}( & \left.K^{i \bar{j}} D_{i} W D_{\bar{j}} \bar{W}-3|W|^{2}\right) \\
& +\frac{1}{2} \sum_{a, b} f_{a b}\left(q_{i}^{(a)} \phi^{i} K_{i}+\xi_{a}\right)\left(q_{j}^{(b)} \phi^{j} K_{j}+\xi_{b}\right)
\end{aligned}
$$

where we have temporarily set $M_{P}=1$ and where we have employed the usual notation $K_{i} \equiv \partial_{i} K, K_{i \bar{j}} \equiv \partial_{i} \partial_{\bar{j}} K$, and $F_{i} \equiv D_{i} W=\partial_{i} W+K_{i} W$. Here $f_{a b}$ are the gauge kinetic functions of the Abelian gauge groups, which depend on the moduli fields which we are implicitly assuming to be stabilized. Of course, after moduli stabilization, we should be considering gauged $R$-symmetries at the supergravity level [15]. However, since our main focus is on the scalar potential, this subtlety does not affect the present discussion and it is enough to consider the expectation value $\left\langle f_{a b}\right\rangle=\delta_{a b} / g_{a}^{2}$. Note that the gravitino mass is $m_{3 / 2}^{2}=|W|^{2} \exp (K)$.

As before, the stability of a given extremum in the supergravity framework depends on the scalar mass matrix: an extremum represents a stable vacuum or flat direction only if the mass matrix has no negative eigenvalues. Of a particular importance for our purposes are the $D$-term contributions to the scalar masses, which 
after moduli stabilization are given by

$$
\left\{\begin{array}{l}
\partial_{i} \partial_{\bar{j}} V_{D}=g_{a}^{2} q_{i}^{(a)}\left(K_{\bar{j}}^{i}+\bar{\phi}^{\bar{l}} K_{\bar{j} \bar{l}}^{i}\right) D_{a}+Q_{i k} \phi^{k} \bar{\phi}^{\bar{l}} K_{\bar{l}}^{i} K_{\bar{j}}^{k} \\
\partial_{i} \partial_{j} V_{D}=g_{a}^{2} q_{i}^{(a)} \bar{\phi}^{\bar{l}} K_{j \bar{l}}^{i} D_{a}+Q_{i j} \bar{\phi}^{\bar{l}} K_{\bar{l}}^{i} K^{j}+Q_{i k} \phi^{k} \bar{\phi}^{\bar{l}} K_{\bar{l}}^{i} K_{\bar{j}}^{k}
\end{array}\right.
$$

where the charge matrix $Q_{i j}$ is defined as

$$
Q_{i j} \equiv \sum_{a=1}^{n} q_{i}^{(a)} q_{j}^{(a)}
$$

The supergravity effects on the $U(1)^{n}$ deconstruction model described in the previous section can be easily described in the limit in which the supergravity moduli fields are heavy and can be integrated out. This leads to a low-energy theory with soft breaking terms. It is clear from gauge invariance that the resulting soft breaking terms are of the form

$$
V_{\mathrm{soft}}=\sum_{i=1}^{n+1} \tilde{m}_{i}^{2}\left|\phi_{i}\right|^{2}+\left\{A \lambda \prod_{i=1}^{n+1} \phi_{i}+\text { h.c. }\right\} .
$$

The $A$-term induced by the Wilson-line superpotential has a negligible effect on the dynamics for large $n$, and can safely be neglected in the following. However, the effects of the soft terms $\tilde{m}_{i}^{2}$ on the vacua described in previous sections are quite clear. Specifically, just as we saw in previous sections, the large number of flux vacua which were previously unstable become now stable in a certain region of the parameter space $\left(\xi_{1}, \xi_{n}\right)$.

Clearly, this method of increasing the number of stable vacua only works for large soft masses, of the order of the FI terms. From this point of view, a large supersymmetry breaking scale stabilizing most of the landscape vacua fits well with the "split supersymmetry" proposal in Ref. [16], in which a large supersymmetrybreaking scale was assumed to go together with the presence of a large number of vacua.

\subsection{A supergravity toy model and the cosmological constant problem}

In order to quantify the issue of the cancellation of the cosmological constant in a simpler setting, we shall now propose a simple supergravity model, inspired by the toy models discussed in the previous sections and by the flux compactifications of string theory [6]. Since the deconstruction model of Sect. 3 is difficult to analyze in this context, we will instead consider the supergravity extension of $n$ copies of the original FI model. Thus, our model will be based on the gauge group $U(1)^{n}$ and chiral superfields $\Phi_{i}^{(+)}, \Phi_{i}^{(-)}$with charges \pm 1 under the gauge group factor $U(1)_{i}$. These fields are therefore coupled to each other only through supergravity interactions. 
The model has a single Kähler modulus $T$ describing the volume of a Calabi-Yau or orbifold compactification, and has supergravity potentials described by

$$
\begin{aligned}
K & =-3 \ln \left(T+\bar{T}-\sum_{i=1}^{n}\left|\Phi_{i}^{(+)}\right|^{2}-\sum_{i=1}^{n}\left|\Phi_{i}^{(-)}\right|^{2}\right) \\
W & =\sum_{i=1}^{n} m_{i} \Phi_{i}^{(+)} \Phi_{i}^{(-)}+W_{0}+h e^{-b_{0} T}
\end{aligned}
$$

where $W_{0}$ is determined by the fluxes according to Ref. [6] and the nonperturbative (exponential) term in the superpotential, responsible for the stabilization of the Kähler modulus T, could come from D7-branes, gaugino condensation, or Euclidean D3-branes [17]. All other moduli are assumed to be stabilized by other dynamical effects, e.g., by adding appropriate fluxes.

If we first set the FI terms to zero, i.e., $\xi_{i}=0$, there is a supersymmetric solution giving an AdS space, obtained by solving $F_{T}=0$. However, in the presence of the FI terms, the vev's of the moduli fields will be changed. If the FI terms and the vev's of the fields $\phi_{i}^{( \pm)}$are sufficiently small compared to the Planck scale, we can effectively integrate out the $T$-field. This will give rise to explicit soft terms in the Lagrangian [18]. The part of the scalar potential which is relevant for discussing the cosmological constant in the various vacua of the theory is then given by

$$
\begin{gathered}
V=\sum_{i=1}^{n}\left[\left(m_{i}^{2}+\tilde{m}_{i}^{2}\right)\left(\left|\phi_{i}^{(+)}\right|^{2}+\left|\phi_{i}^{(-)}\right|^{2}\right)+B_{i}\left(\phi_{i}^{(+)} \phi_{i}^{(-)}+\bar{\phi}_{i}^{(+)} \bar{\phi}_{i}^{(-)}\right)\right] \\
+\sum_{i=1}^{n} \frac{g_{i}^{2}}{2}\left(\left|\phi_{i}^{(+)}\right|^{2}-\left|\phi_{i}^{(-)}\right|^{2}+\xi_{i}\right)^{2}-3 m_{3 / 2}^{2} M_{P}^{2}
\end{gathered}
$$

where $\tilde{m}_{i}^{2}$ and $B_{i}$ describe the soft terms and where we have assumed equal soft masses for the fields $\phi_{i}^{(+)}$and $\phi_{i}^{(-)}$for simplicity.

A few words of caution are in order here. Note that the potential (5.6) has been written to the lowest order in the matter fields $\phi_{i}^{( \pm)}$. Thus, for example, the gravitino mass, which is a function of all the fields, becomes a constant. As a result of these approximations, the vacua derived from Eq. (5.6) are certainly only approximations to the true vacua. Note, in addition, that we have also neglected perturbative loop effects as well as non-perturbative effects. Moreover, integrating out the field $T$ in Eq. (5.5) is a good approximation only if the field $T$ is heavier than the charged fields $\phi_{i}$. This is not always a good approximation to the dynamics of the model. Despite these issues, we believe that our discussion concerning the possible cancellation of the cosmological constant will still be of qualitative value, and that the qualitative features of such a cancellation - which relies on balancing the positive contributions to the vacuum energy coming from the supersymmetry-breaking against the negative contributions coming from the supergravity interactions - capture the qualitative features of the full quantum problem. 
Minimizing the scalar potential in Eq. (5.6), we find $3^{n}$ possible vacua, determined as simultaneous solutions of the coupled equations

$$
\begin{aligned}
& \left(m_{i}^{2}+\tilde{m}_{i}^{2}+g_{i}^{2} D_{i}\right) v_{i}^{(+)}+B_{i} \bar{v}_{i}^{(-)}=0 \\
& \left(m_{i}^{2}+\tilde{m}_{i}^{2}-g_{i}^{2} D_{i}\right) v_{i}^{(-)}+B_{i} \bar{v}_{i}^{(+)}=0
\end{aligned}
$$

where $v_{i}^{( \pm)} \equiv\left\langle\phi_{i}^{( \pm)}\right\rangle$. Indeed, the field equations (5.7) have three solutions for each $i$. By performing $U(1)$ rotations, the solutions can always be made real.

First, it is useful to define

$$
m_{S, i}^{2} \equiv m_{i}^{2}+\tilde{m}_{i}^{2}, \quad \Delta_{i}^{2} \equiv \sqrt{m_{S, i}^{4}-B_{i}^{2}}
$$

for notational simplicity. We then find that the first class of solutions are given by

$$
\left|v_{i}^{(+)}\right|^{2}=-\frac{\left(\Delta_{i}^{2}+m_{S, i}^{2}\right)\left(\Delta_{i}^{2}+g_{i}^{2} \xi_{i}\right)}{2 g_{i}^{2} \Delta_{i}^{2}}, \quad\left|v_{i}^{(-)}\right|^{2}=\frac{\left(\Delta_{i}^{2}-m_{S, i}^{2}\right)\left(\Delta_{i}^{2}+g_{i}^{2} \xi_{i}\right)}{2 g_{i}^{2} \Delta_{i}^{2}}
$$

which leads to $D$-terms and vacuum energy $\Lambda_{0}$ of the form

$$
g_{i}^{2}\left\langle D_{i}\right\rangle=-\Delta_{i}^{2}, \quad \Lambda_{0}=\sum_{i} \frac{\Delta_{i}^{4}}{g_{i}^{2}}\left(-\frac{1}{2}-\frac{g_{i}^{2} \xi_{i}}{\Delta_{i}^{2}}\right)-3 m_{3 / 2}^{2} M_{P}^{2} .
$$

The second class of solutions, by contrast, are given by

$$
\left|v_{i}^{(+)}\right|^{2}=\frac{\left(\Delta_{i}^{2}-m_{S, i}^{2}\right)\left(\Delta_{i}^{2}-g_{i}^{2} \xi_{i}\right)}{2 g_{i}^{2} \Delta_{i}^{2}}, \quad\left|v_{i}^{(-)}\right|^{2}=-\frac{\left(\Delta_{i}^{2}+m_{S, i}^{2}\right)\left(\Delta_{i}^{2}-g_{i}^{2} \xi_{i}\right)}{2 g_{i}^{2} \Delta_{i}^{2}},
$$

which lead to

$$
g_{i}^{2}\left\langle D_{i}\right\rangle=\Delta_{i}^{2}, \quad \Lambda_{0}=\sum_{i} \frac{\Delta_{i}^{4}}{g_{i}^{2}}\left(-\frac{1}{2}+\frac{g_{i}^{2} \xi_{i}}{\Delta_{i}^{2}}\right)-3 m_{3 / 2}^{2} M_{P}^{2} .
$$

Clearly these solutions exist only when $\left|v_{i}^{( \pm)}\right|^{2}>0$, which means that $g_{i}^{2}\left|\xi_{i}\right|>\Delta^{2}$ and $m_{S, i}^{2}>\Delta^{2}$ where $\xi_{i}<0$ for Eq. (5.10) and $\xi_{i}>0$ for Eq. (5.12). One can also verify that these solutions are stable. Finally, the third class of solutions is trivially

$$
v_{i}^{(+)}=v_{i}^{(-)}=0
$$

which is easily verified to be stable if

$$
m_{S, i}^{2} \pm \sqrt{B_{i}^{2}+g_{i}^{4} \xi_{i}^{2}} \geq 0
$$

Of course, in the limit of vanishing soft terms, i.e., $\tilde{m}_{i}^{2}, B_{i} \rightarrow 0$, all three classes of solutions reduce to the standard global minima of the Fayet-Iliopoulos model discussed in Sect. 2. 
From a string-theory perspective, the limit of interest corresponds to taking $\left|\xi_{i}\right| \gg$ $m_{i}^{2}, \tilde{m}_{i}^{2}, B_{i}$. In this limit, the cancellation of the cosmological constant then takes the approximate form

$$
\sum_{i=1}^{n}\left|\xi_{i}\right| \sqrt{\left(m_{i}^{2}+\tilde{m}_{i}^{2}\right)^{2}-B_{i}^{2}} \simeq 3 m_{3 / 2}^{2} M_{P}^{2},
$$

for the $2^{n}$ vacua in the classes in Eqs. (5.10) and (5.12). In gravity-mediated supersymmetry breaking scenarios such as we are considering, we expect $\tilde{m}_{i}^{2}, B_{i} \sim m_{3 / 2}^{2}$. Since typical values for the Fayet-Iliopoulos coefficients $\xi_{i}$ are smaller by one or two orders of magnitude compared to the Planck scale, we see that there are therefore basically only two possible ways in which these $2^{n}$ vacua can yield a small cosmological constant:

- We can have $m_{i}^{2} \gg m_{3 / 2}^{2}$. However, in this case, partial cancellation can occur only for a small number of gauge factors, which means that a reasonable accommodation for a small cosmological constant is difficult to achieve.

- Alternatively, we can have $m_{i}^{2} \leq m_{3 / 2}^{2}$. In this case, the cosmological constant can be cancelled by combining the two different types of vacua in Eqs. (5.10) and (5.12). For example, we would require $n \sim 10-100$ for generic values $\xi_{i} \sim\left(10^{-1}-10^{-2}\right) M_{P}^{2}$. Indeed, in such cases, there will be a large number of landscape vacua with small vacuum energies. Of course, any vacuum solutions which also contain the third class of solutions in Eq. (5.13) will add contributions $\frac{1}{2} g_{j}^{2} \xi_{j}^{2}$ to the vacuum energy $\Lambda_{0}$. These contributions are very large, and will upset the cancellation of the predicted cosmological constant unless $\left|\xi_{j}\right| \ll m_{3 / 2} M_{P}$

By contrast, in the opposite limit of small FI terms, cancellation occurs for typical values of soft terms $\tilde{m}^{2} \sim \sqrt{3} m_{3 / 2} M_{P} / n$.

The outcome of this discussion, then, is that it is the second possibility itemized above which can best accommodate a small cosmological constant in our toy model. Barring additional fine-tunings, this clearly points towards requiring $m_{i}^{2} \ll \tilde{m}_{i}^{2}$, from which we conclude that even a qualitative supergravity analysis of the vacuum structure of any landscape model is crucial for addressing the smallness of the cosmological constant.*

High-scale supersymmetry breaking therefore seems to have two positive features in our $D$-term landscape models. First, they render most of the vacua stable by adding large diagonal soft masses. Second, they allow a possible cancellation of the cosmological constant.

*Of course, in addition to the stability of the vacua and the fine-tuning of the cosmological constant (both of which seem to favor large soft masses), a second crucial fine-tuning in this case is the light Higgs mass. This can only be addressed within the context of a specific model in which Standard Model or MSSM fields are coupled to the $U(1)$ fields we are investigating, and is thus beyond the scope of the current paper. 


\section{Discussion}

In this paper, we proposed a field-theoretic framework giving rise to models containing large numbers of vacuum solutions. The field-theoretic nature of these models therefore allowed us to explicitly calculate such quantities of interest as the ratio of stable versus total numbers of vacua, the number of $R$-symmetry preserving vacua, and the supersymmetry-breaking scale. While at the field-theory level many models with large numbers of vacua can certainly be given, our examples have the advantage of describing large classes of string compactifications with Abelian gauge groups and FI terms. Moreover, within this large class, we presented specific examples involving discretized versions of magnetic fluxes in the internal space, as obtained by deconstructing (supersymmetric) models with $U(1)$ gauge fields on the orbifold $S^{1} / \mathbb{Z}_{2}$. By examining the extrema involving vanishing vev's for bi-fundamental fields, we found that these solutions correspond to profiles which are linear in $x_{5}$ for the odd-scalar $\Sigma=A_{6}$ in the five-dimensional vector multiplet. These solutions therefore correspond to constant magnetic fluxes $\partial_{5} \Sigma=F_{56}$ from a six-dimensional perspective. The number of deconstructed magnetic fluxes is large, and although they all have Nielsen-Olesen instabilities, they can be stabilized by $F$-term contributions coming from supergravity interactions. This therefore generates a field-theory landscape which is similar in spirit and closely related to the landscapes currently under discussion in string-theory contexts [2]. We also showed that (super)gravity effects turn out to be consistent with (and actually needed for) a qualitative accommodation of the smallness of the cosmological constant.

One of the interesting results of the landscape picture emerging in the class of models we considered in this paper is the possibility of passing from one vacuum to another by renormalization group flow. This possibility arises because our fundamental defining parameters, such as $\xi_{i}$ and $\lambda$, can change with the energy scale. We showed in an explicit toy model that this renormalization group flow can indeed induce boundary crossings. A general feature of the boundary separating two different vacua is the presence of a massless scalar in the spectrum, which suggests an interpretation in terms of phase transitions. This type of boundary crossing could therefore be of potential interest for inflationary models. Moreover, the gauge symmetry-breaking pattern can also change as a result of boundary crossings. The corresponding phase transitions could therefore also be relevant for electroweak symmetry-breaking in the early Universe and for baryogenesis. We hope to return to some of these interesting phenomena in the future.

The example(s) that we analyzed in some detail in this paper were motivated by providing a purely four-dimensional description of higher-dimensional fluxes, in the spirit of deconstruction. There are clearly several possible generalizations of this setup. One possibility would be to add flavors to the models discussed in Sect. 3, again in the spirit of deconstruction. Note that the net effect of adding such flavors is to add new fields. For example, if we add flavors in the bulk [i.e., at all $U(1)$ sites 
except the first and last], the total number of fields for the $U(1)^{n}$ model would grow to $3(n-1)$. The naive number of extrema in this case would then be $\sim \mathcal{O}\left(2^{3 n-4}\right)$ but may actually be smaller in practice. There are also new $F$-term contributions to the scalar potential which render some of these vacua stable.

The field-theoretic framework proposed in this paper also allowed us to explicitly calculate the ratio of stable vacua to the total numbers of extrema. We found that this ratio is much larger than a naïve estimate based on random sign assignments for mass eigenvalues would suggest. We also found in Sect. 3 that these sorts of results - and indeed the entire vacuum structure of the models we considered - are very sensitive not only to dimensionful mass parameters, such as FI terms and various type of scalar masses (all of which are natural landscape coordinates), but also to dimensionless parameters such as the Yukawa and gauge couplings.

There are clearly more general examples that can arise in string models with Dbranes. However, these will depend on the particular classes of string models under consideration. For example, in heterotic string constructions, there is at most one anomalous $U(1)$ factor, with the universal axion mixing à la Stückelberg [19]. By contrast, in a Type I string model (or equivalently a Type II orientifold), there can be a large number of anomalous $U(1)$ factors, each equipped with its own FI term [20].

One natural framework to consider consists of taking a large number of $U(1)$ 's, each with its FI term, and all possible charged bi-fundamental fields. If there are $n$ different $U(1)$ gauge factors, this would imply $n(n+1) / 2$ bi-fundamental fields. Such a setup would emerge naturally in Type I or Type II string models containing large numbers of $U(1)$ D-branes which either sit at the same point in the compact space or intersect each other. In such a geometry, the bi-fundamental fields would emerge as open-string excitations stretching from one $U(1)$ brane to another. This setup would not only yield a large number of vacua, but would also naturally give rise to various new types of superpotential terms. These include cubic, quartic, and higher-order interactions, each of which would produce additional $F$-term contributions to the scalar potential. It is easy to imagine that in some region of the parameter space, these additional contributions could easily stabilize most of the huge numbers of vacua that such models would produce.

Regardless of the particular field-theoretic model under discussion, it is apparent that string models of all sorts generically give rise to multiple $U(1)$ gauge factors. We therefore believe that even though the models we presented in this paper are fieldtheoretic in nature, they will emerge naturally in realistic string contexts and should be viewed, quite literally, as at least one component of the full string landscape. As such, we believe that these types of models should be analyzed further, as they undoubtedly play a direct role in affecting the statistical properties of string vacua. 


\section{Acknowledgments}

KRD is supported in part by the US National Science Foundation under Grant PHY/0301998, by the US Department of Energy under Grant DE-FG02-04ER41298, and by a Research Innovation Award from Research Corporation. ED is supported in part by the CNRS PICS \#2530, INTAS grant 03-51-6346, the RTN grants MRTN-CT-2004-503369 and MRTN-CT-2004-005104, and by a European Union Excellence Grant, MEXT-CT-2003-509661. TG is supported in part by a US Department of Energy grant DE-FG02-94ER-40823, a grant from the Office of the Dean of the Graduate School of the University of Minnesota, and a Research Innovation Award from Research Corporation. KRD and ED would like to acknowledge the hospitality of the William I. Fine Theoretical Physics Institute at the University of Minnesota, and KRD also wishes to acknowledge the hospitality of both CERN and the CPhT-Ecole Polytechnique. We also wish to acknowledge the hospitality of the Aspen Center for Physics where this work was initiated.

\section{Appendix}

In this Appendix we present the details behind the $n=3$ landscape discussed in Sect. 4.1 and sketched in Fig. 11. As discussed in Sect. 4.1, we shall set $\lambda=0$ and take $\tilde{m}^{2} \geq 0$. The surviving extrema are then in the following classes:

$\{\emptyset\}$ : These are extrema with all $v_{i} \equiv\left\langle\phi_{i}\right\rangle=0$. The eigenvalues are:

$$
\tilde{m}^{2} \pm \xi_{1} \quad(2), \quad \tilde{m}^{2} \pm \xi_{3} \quad(2),
$$

where the number in parentheses corresponds to the multiplicity of the eigenvalues. Thus, provided that

$$
\left|\xi_{1}\right|<\tilde{m}^{2} \text { and }\left|\xi_{3}\right|<\tilde{m}^{2},
$$

this vacuum is stable. Clearly, when $\tilde{m}=0$ this vacuum is unstable. The $D$-terms are given by

$$
\langle\vec{D}\rangle=\left\{\xi_{1}, 0, \xi_{3}\right\} .
$$

$\{1\}:\left|v_{1}\right|^{2}=\xi_{1}-\tilde{m}^{2}, v_{2}=v_{3}=v_{4}=0$, with eigenvalues

$$
0, \quad 2\left(\xi_{1}-\tilde{m}^{2}\right), \quad 2 \tilde{m}^{2}(2), \quad \tilde{m}^{2} \pm \xi_{3} \quad(2) .
$$

The stability region is:

$$
\xi_{1}>\tilde{m}^{2} \text { and }\left|\xi_{3}\right|<\tilde{m}^{2} .
$$

When $\tilde{m}=0$ this vacuum is no longer stable. The $D$-terms are given by

$$
\langle\vec{D}\rangle=\left\{\tilde{m}^{2}, 0, \xi_{3}\right\} .
$$


$\{2\}:\left|v_{2}\right|^{2}=-\frac{1}{2}\left(\xi_{1}+\tilde{m}^{2}\right), v_{1}=v_{3}=v_{4}=0$, with eigenvalues

$$
0, \quad-2\left(\xi_{1}+\tilde{m}^{2}\right), \quad \frac{1}{2}\left(3 \tilde{m}^{2}-\xi_{1}\right) \quad(2), \quad \frac{1}{2}\left(3 \tilde{m}^{2}+\xi_{1}-2 \xi_{3}\right) \quad(2), \quad \tilde{m}^{2}+\xi_{3}
$$

The stability region is:

$$
\xi_{1}<-\tilde{m}^{2}, \quad \xi_{3}>-\tilde{m}^{2}, \quad \text { and } \quad 2 \xi_{3}-\xi_{1}-3 \tilde{m}^{2}<0 .
$$

When $\tilde{m}=0$ this vacuum is no longer stable. The $D$-terms are given by

$$
\langle\vec{D}\rangle=\left\{\frac{1}{2}\left(\xi_{1}-\tilde{m}^{2}\right), \frac{1}{2}\left(\xi_{1}+\tilde{m}^{2}\right), \xi_{3}\right\}
$$

$\{3\}:\left|v_{3}\right|^{2}=\frac{1}{2}\left(\xi_{3}-\tilde{m}^{2}\right), v_{1}=v_{2}=v_{4}=0$, with eigenvalues

$$
0, \quad 2\left(\xi_{3}-\tilde{m}^{2}\right), \quad \frac{1}{2}\left(3 \tilde{m}^{2}+\xi_{3}\right) \quad(2), \quad \frac{1}{2}\left(3 \tilde{m}^{2}+2 \xi_{1}-\xi_{3}\right) \quad(2), \quad \tilde{m}^{2}-\xi_{1}
$$

The stability region is:

$$
\xi_{1}<\tilde{m}^{2}, \quad \xi_{3}>\tilde{m}^{2}, \quad \text { and } \quad \xi_{3}-2 \xi_{1}-3 \tilde{m}^{2}<0 .
$$

When $\tilde{m}=0$ this vacuum is no longer stable. The $D$-terms are given by

$$
\langle\vec{D}\rangle=\left\{\xi_{1}, \frac{1}{2}\left(\xi_{3}-\tilde{m}^{2}\right), \frac{1}{2}\left(\xi_{3}+\tilde{m}^{2}\right)\right\}
$$

$\{4\}:\left|v_{4}\right|^{2}=-\xi_{3}-\tilde{m}^{2}, v_{1}=v_{2}=v_{3}=0$, with eigenvalues

$$
0, \quad-2\left(\xi_{3}+\tilde{m}^{2}\right), \quad 2 \tilde{m}^{2}(2), \quad \tilde{m}^{2} \pm \xi_{1} \quad(2)
$$

The stability region is:

$$
\xi_{3}<-\tilde{m}^{2} \text { and }\left|\xi_{1}\right|<\tilde{m}^{2}
$$

When $\tilde{m}=0$ this vacuum is no longer stable. The $D$-terms are given by

$$
\langle\vec{D}\rangle=\left\{\xi_{1}, 0,-\tilde{m}^{2}\right\}
$$

$\{12\}$ : There are no solutions in this class.

$\{13\}:\left|v_{1}\right|^{2}=\xi_{1}-\tilde{m}^{2},\left|v_{3}\right|^{2}=\frac{1}{2}\left(\xi_{3}-\tilde{m}^{2}\right), v_{2}=v_{4}=0$, with eigenvalues

$$
0(2), \quad 2\left(\xi_{1}-\tilde{m}^{2}\right), \quad 2\left(\xi_{3}-\tilde{m}^{2}\right), \quad \frac{1}{2}\left(5 \tilde{m}^{2}-\xi_{3}\right) \quad(2), \quad \frac{1}{2}\left(3 \tilde{m}^{2}+\xi_{3}\right) \quad(2) .
$$

The stability region is

$$
\xi_{1}>\tilde{m}^{2}, \quad \text { and } \quad \tilde{m}^{2}<\xi_{3}<5 \tilde{m}^{2}
$$


When $\tilde{m}=0$ this vacuum is no longer stable. The $D$-terms are given by

$$
\langle\vec{D}\rangle=\left\{\tilde{m}^{2}, \frac{1}{2}\left(\xi_{3}-\tilde{m}^{2}\right), \frac{1}{2}\left(\xi_{3}+\tilde{m}^{2}\right)\right\} .
$$

$\{14\}:\left|v_{1}\right|^{2}=\xi_{1}-\tilde{m}^{2},\left|v_{4}\right|^{2}=-\xi_{3}-\tilde{m}^{2}, v_{2}=v_{3}=0$, with eigenvalues

$$
0(2), \quad 2\left(\xi_{1}-\tilde{m}^{2}\right), \quad-2\left(\xi_{3}+\tilde{m}^{2}\right), \quad 2 \tilde{m}^{2}(4) .
$$

The stability region is

$$
\xi_{1}>\tilde{m}^{2} \text { and } \xi_{3}<-\tilde{m}^{2} .
$$

When $\tilde{m}=0$ this vacuum becomes supersymmetric. The $D$-terms are given by

$$
\langle\vec{D}\rangle=\left\{\tilde{m}^{2}, 0,-\tilde{m}^{2}\right\}
$$

$\{23\}:\left|v_{2}\right|^{2}=\frac{1}{3}\left(-3 \tilde{m}^{2}-2 \xi_{1}+\xi_{3}\right),\left|v_{3}\right|^{2}=\frac{1}{3}\left(-3 \tilde{m}^{2}-\xi_{1}+2 \xi_{3}\right), v_{1}=v_{4}=0$, with eigenvalues

$$
\begin{aligned}
& 0(2), \quad \frac{1}{3}\left(6 \tilde{m}^{2} \pm \xi_{1} \pm \xi_{3}\right) \quad(2), \\
& -4 \tilde{m}^{2}-2 \xi_{1}+2 \xi_{3} \pm \frac{2}{\sqrt{3}} \sqrt{3 \tilde{m}^{4}+3 \tilde{m}^{2}\left(\xi_{1}-\xi_{3}\right)+\xi_{1}^{2}-\xi_{1} \xi_{3}+\xi_{3}^{2}}
\end{aligned}
$$

The stability region is

$$
\xi_{1}<2 \xi_{3}-3 \tilde{m}^{2}, \quad \xi_{3}>2 \xi_{1}+3 \tilde{m}^{2}, \quad \text { and } \quad\left|\xi_{1}+\xi_{3}\right|<6 \tilde{m}^{2} .
$$

When $\tilde{m}=0$ this vacuum is no longer stable. The $D$-terms are given by

$$
\langle\vec{D}\rangle=\left\{-\tilde{m}^{2}+\frac{1}{3}\left(\xi_{1}+\xi_{3}\right), \frac{1}{3}\left(\xi_{1}+\xi_{3}\right), \tilde{m}^{2}+\frac{1}{3}\left(\xi_{1}+\xi_{3}\right)\right\}
$$

$\{24\}:\left|v_{2}\right|^{2}=\frac{1}{2}\left(-\xi_{1}-\tilde{m}^{2}\right),\left|v_{4}\right|^{2}=-\xi_{3}-\tilde{m}^{2}, v_{1}=v_{3}=0$, with eigenvalues

$$
0(2), \quad-2\left(\xi_{1}+\tilde{m}^{2}\right), \quad-2\left(\xi_{3}+\tilde{m}^{2}\right), \quad \frac{1}{2}\left(5 \tilde{m}^{2}+\xi_{1}\right)(2), \quad \frac{1}{2}\left(3 \tilde{m}^{2}-\xi_{1}\right)
$$

The stability region is

$$
-5 \tilde{m}^{2}<\xi_{1}<-\tilde{m}^{2} \text { and } \xi_{3}<-\tilde{m}^{2} \text {. }
$$

When $\tilde{m}=0$ this vacuum is no longer stable. The $D$-terms are given by

$$
\langle\vec{D}\rangle=\left\{\frac{1}{2}\left(\xi_{1}-\tilde{m}^{2}\right), \frac{1}{2}\left(\xi_{1}+\tilde{m}^{2}\right),-\tilde{m}^{2}\right\} .
$$

$\{34\}$ : There are no solutions in this class. 
$\{123\}:\left|v_{1}\right|^{2}=\xi_{1}+\xi_{3}-6 \tilde{m}^{2},\left|v_{2}\right|^{2}=\xi_{3}-5 \tilde{m}^{2},\left|v_{3}\right|^{2}=\xi_{3}-3 \tilde{m}^{2}, v_{4}=0$. Some of the eigenvalue expressions are quite complicated but one can verify that the stability region is:

$$
\xi_{1}+\xi_{3}>6 \tilde{m}^{2}, \quad \text { and } \quad \xi_{3}>5 \tilde{m}^{2}
$$

When $\tilde{m}=0$ this vacuum remains stable. The $D$-terms are given by

$$
\langle\vec{D}\rangle=\left\{\tilde{m}^{2}, 2 \tilde{m}^{2}, 3 \tilde{m}^{2}\right\}
$$

$\{\mathbf{1 2 4}\}$ : There are no solutions in this class.

$\{134\}$ : There are no solutions in this class.

$\{234\}:\left|v_{2}\right|^{2}=-\xi_{1}-3 \tilde{m}^{2},\left|v_{3}\right|^{2}=-\xi_{1}-5 \tilde{m}^{2},\left|v_{4}\right|^{2}=-\xi_{1}-\xi_{3}-6 \tilde{m}^{2}, v_{1}=0$. Some of the eigenvalue expressions are quite complicated but one can verify that the stability region is:

$$
\xi_{1}+\xi_{3}<-6 \tilde{m}^{2} \text { and } \xi_{1}<-5 \tilde{m}^{2}
$$

When $\tilde{m}=0$ this vacuum remains stable. The $D$-terms are given by

$$
\langle\vec{D}\rangle=\left\{-3 \tilde{m}^{2},-2 \tilde{m}^{2},-\tilde{m}^{2}\right\} \text {. }
$$

$\{1234\}$ : There are no solutions in this class unless $\tilde{m}=0$. For $\tilde{m}=0$, we have a line of solutions given by

$$
\left|v_{1}\right|^{2}=\left|v_{3}\right|^{2}+\xi_{1}, \quad\left|v_{4}\right|^{2}=\left|v_{3}\right|^{2}-\xi_{3}, \quad\left|v_{2}\right|^{2}=\left|v_{3}\right|^{2} .
$$

These solutions are necessarily supersymmetric, with all $D$-terms vanishing.

For $\tilde{m}^{2}<0$, we obtain solutions for the remaining cases:

$\{12\}:\left|v_{1}\right|^{2}=\xi_{1}-3 \tilde{m}^{2},\left|v_{2}\right|^{2}=-2 \tilde{m}^{2}, v_{3}=v_{4}=0$, with eigenvalues

$0(2), \quad \tilde{m}^{2}+\xi_{3}(2), \quad 3 \tilde{m}^{2}-\xi_{3}(2), \quad-7 \tilde{m}^{2}+\xi_{1} \pm \sqrt{25 \tilde{m}^{4}-6 \xi_{1} \tilde{m}^{2}+\xi_{1}^{2}}$.

This vacuum has no stable regions, and the $D$-terms are given by

$$
\langle\vec{D}\rangle=\left\{\tilde{m}^{2}, 2 \tilde{m}^{2}, \xi_{3}\right\}
$$

$\{34\}:\left|v_{3}\right|^{2}=-2 \tilde{m}^{2},\left|v_{4}\right|^{2}=-\xi_{3}-3 \tilde{m}^{2}, v_{1}=v_{2}=0$, with eigenvalues

$0(2), \quad \tilde{m}^{2}-\xi_{1}(2), \quad 3 \tilde{m}^{2}+\xi_{1}(2), \quad-7 \tilde{m}^{2}-\xi_{3} \pm \sqrt{25 \tilde{m}^{4}+6 \tilde{m}^{2} \xi_{3}+\xi_{3}^{2}}$.

This vacuum has no stable regions, and the $D$-terms are given by

$$
\langle\vec{D}\rangle=\left\{\xi_{1},-2 \tilde{m}^{2},-\tilde{m}^{2}\right\} \text {. }
$$

\{124\}: $\left|v_{1}\right|^{2}=-\xi_{3}-\tilde{m}^{2},\left|v_{2}\right|^{2}=\xi_{1}-3 \tilde{m}^{2},\left|v_{4}\right|^{2}=-2 \tilde{m}^{2}, v_{3}=0$. This vacuum has no stable regions, and the $D$-terms are given by

$$
\langle\vec{D}\rangle=\left\{\tilde{m}^{2}, 2 \tilde{m}^{2},-\tilde{m}^{2}\right\}
$$

$\{134\}:\left|v_{1}\right|^{2}=\xi_{1}-\tilde{m}^{2},\left|v_{3}\right|^{2}=-2 \tilde{m}^{2},\left|v_{4}\right|^{2}=-\xi_{3}-3 \tilde{m}^{2}, v_{2}=0$. This vacuum has no stable regions, and the $D$-terms are given by

$$
\langle\vec{D}\rangle=\left\{\tilde{m}^{2},-2 \tilde{m}^{2},-\tilde{m}^{2}\right\} .
$$




\section{References}

[1] R. Bousso and J. Polchinski, JHEP 0006, 006 (2000) [arXiv:hep-th/0004134]; J. L. Feng, J. March-Russell, S. Sethi and F. Wilczek, Nucl. Phys. B 602, 307 (2001) [arXiv:hep-th/0005276]; for a general review, see R. Bousso and J. Polchinski, Sci. Am. 291, 60 (2004).

[2] L. Susskind, arXiv:hep-th/0302219; M. R. Douglas, JHEP 0305, 046 (2003) [arXiv:hep-th/0303194]; M. Dine, arXiv:hep-th/0410201.

[3] S. Ashok and M. R. Douglas, JHEP 0401, 060 (2004) [arXiv:hep-th/0307049]; M. R. Douglas, B. Shiffman and S. Zelditch, Commun. Math. Phys. 252, 325 (2004) [arXiv:math.cv/0402326]. F. Denef and M. R. Douglas, JHEP 0405, 072 (2004) [arXiv:hep-th/0404116]; A. Giryavets, S. Kachru and P. K. Tripathy, JHEP 0408, 002 (2004) [arXiv:hep-th/0404243]; M. R. Douglas, B. Shiffman and S. Zelditch, arXiv:math.cv/0406089; O. DeWolfe, A. Giryavets, S. Kachru and W. Taylor, arXiv:hep-th/0411061; J. P. Conlon and F. Quevedo, JHEP 0410, 039 (2004) [arXiv:hep-th/0409215]; F. Denef and M. R. Douglas, arXiv:hep-th/0411183; R. Blumenhagen, F. Gmeiner, G. Honecker, D. Lust and T. Weigand, arXiv:hep-th/0411173.

[4] T. Banks, M. Dine and E. Gorbatov, JHEP 0408, 058 (2004) [arXiv:hepth/0309170]; M. R. Douglas, arXiv:hep-th/0405279; D. Robbins and S. Sethi, arXiv:hep-th/0405011; H. Firouzjahi, S. Sarangi and S. H. H. Tye, JHEP 0409, 060 (2004) [arXiv:hep-th/0406107]; X. Calmet, arXiv:hep-ph/0406314; E. Silverstein, arXiv:hep-th/0407202; B. Freivogel and L. Susskind, arXiv:hepth/0408133; J. Kumar and J. D. Wells, arXiv:hep-th/0409218; G. Dvali, arXiv:hep-th/0410286; J. D. Wells, arXiv:hep-ph/0411041; R. Kallosh and A. Linde, arXiv:hep-th/0411011.

[5] S. Weinberg, Phys. Rev. Lett. 59, 2607 (1987); A. Vilenkin, Phys. Rev. Lett. 74, 846 (1995) [arXiv:gr-qc/9406010]; V. Agrawal, S. M. Barr, J. F. Donoghue and D. Seckel, Phys. Rev. Lett. 80, 1822 (1998) [arXiv:hep-ph/9801253]; M. L. Graesser, S. D. H. Hsu, A. Jenkins and M. B. Wise, Phys. Lett. B 600, 15 (2004) [arXiv:hep-th/0407174]; A. Vilenkin, arXiv:astro-ph/0407586.

[6] S. B. Giddings, S. Kachru and J. Polchinski, Phys. Rev. D 66, 106006 (2002) [arXiv:hep-th/0105097]; K. Becker and M. Becker, JHEP 0107, 038 (2001) [arXiv:hep-th/0107044]; K. Becker, M. Becker, M. Haack and J. Louis, JHEP 0206, 060 (2002) [arXiv:hep-th/0204254]; S. Kachru， M. B. Schulz and S. Trivedi, JHEP 0310, 007 (2003) [arXiv:hep-th/0201028]; S. Kachru, R. Kallosh, A. Linde and S. P. Trivedi, Phys. Rev. D 68, 046005 (2003) [arXiv:hep-th/0301240]; R. Blumenhagen, D. Lust and T. R. Taylor, Nucl. Phys. B 663, 319 (2003) [arXiv:hep-th/0303016]; J. F. G. Cascales and 
A. M. Uranga, JHEP 0305, 011 (2003) [arXiv:hep-th/0303024]; P. G. Camara, L. E. Ibanez and A. M. Uranga, arXiv:hep-th/0408036; M. Grana, T. W. Grimm, H. Jockers and J. Louis, Nucl. Phys. B 690, 21 (2004) [arXiv:hepth/0312232]. F. Marchesano and G. Shiu, arXiv:hep-th/0408059; JHEP 0411, 041 (2004) [arXiv:hep-th/0409132]; A. Font, JHEP 0411, 077 (2004) [arXiv:hepth/0410206]; F. Marchesano, G. Shiu and L. T. Wang, arXiv:hep-th/0411080.

[7] N. Arkani-Hamed, A. G. Cohen and H. Georgi, Phys. Rev. Lett. 86, 4757 (2001) [arXiv:hep-th/0104005]; C. T. Hill, S. Pokorski and J. Wang, Phys. Rev. D 64, 105005 (2001) [arXiv:hep-th/0104035].

[8] C. Bachas, arXiv:hep-th/9503030; M. Berkooz, M. R. Douglas and R. G. Leigh, Nucl. Phys. B 480, 265 (1996) [arXiv:hep-th/9606139]; N. Ohta and P. K. Townsend, Phys. Lett. B 418, 77 (1998) [arXiv:hep-th/9710129]; R. Blumenhagen, L. Gorlich and B. Kors, Nucl. Phys. B 569, 209 (2000) [arXiv:hepth/9908130], JHEP 0001, 040 (2000) [arXiv:hep-th/9912204]; G. Pradisi, Nucl. Phys. B 575, 134 (2000) [arXiv:hep-th/9912218]; R. Blumenhagen, L. Goerlich, B. Kors and D. Lust, JHEP 0010,006 (2000) [arXiv:hep-th/0007024]; C. Angelantonj, I. Antoniadis, E. Dudas and A. Sagnotti, Phys. Lett. B 489, 223 (2000) [arXiv:hep-th/0007090]; C. Angelantonj and A. Sagnotti, arXiv:hep-th/0010279; G. Aldazabal, S. Franco, L. E. Ibanez, R. Rabadan and A. M. Uranga, JHEP 0102, 047 (2001) [arXiv:hep-ph/0011132]; M. Cvetic, G. Shiu and A. M. Uranga, Nucl. Phys. B 615, 3 (2001) [arXiv:hep-th/0107166]; C. Kokorelis, JHEP 0209, 029 (2002) [arXiv:hep-th/0205147]; D. Bailin, G. V. Kraniotis and A. Love, Phys. Lett. B 553, 79 (2003) [arXiv:hep-th/0210219]; G. Honecker, Nucl. Phys. B 666, 175 (2003) [arXiv:hep-th/0303015]; M. Larosa and G. Pradisi, Nucl. Phys. B 667, 261 (2003) [arXiv:hep-th/0305224]; G. L. Kane, P. Kumar, J. D. Lykken and T. T. Wang, arXiv:hep-ph/0411125.

[9] P. Fayet and J. Iliopoulos, Phys. Lett. B 51, 461 (1974); P. Fayet, Phys. Lett. B 69, 489 (1977).

[10] L. E. Ibanez, arXiv:hep-ph/0408064; D. Lust, S. Reffert and S. Stieberger, arXiv:hep-th/0410074.

[11] K. R. Dienes, A. E. Faraggi and J. March-Russell, Nucl. Phys. B 467, 44 (1996) [arXiv:hep-th/9510223]; M. Cvetic and P. Langacker, Phys. Rev. D 54, 3570 (1996) [arXiv:hep-ph/9511378]; K. R. Dienes, C. F. Kolda and J. March-Russell, Nucl. Phys. B 492, 104 (1997) [arXiv:hep-ph/9610479]; J. K. Elwood, N. Irges and P. Ramond, Phys. Lett. B 413, 322 (1997) [arXiv:hep-ph/9705270]; B. Kors and P. Nath, arXiv:hep-th/0411201.

[12] E. Dudas, A. Falkowski and S. Pokorski, Phys. Lett. B 568, 281 (2003) [arXiv:hep-th/0303155]; A. Falkowski, H. P. Nilles, M. Olechowski and S. Pokorski, Phys. Lett. B 566, 248 (2003) [arXiv:hep-th/0212206]. 
[13] C. A. Scrucca, M. Serone, L. Silvestrini and F. Zwirner, Phys. Lett. B 525, 169 (2002) [arXiv:hep-th/0110073]; L. Pillo and A. Riotto, Phys. Lett. B 546, 135 (2002) [arXiv:hep-th/0202144]; R. Barbieri, R. Contino, P. Creminelli, R. Rattazzi and C. A. Scrucca, Phys. Rev. D 66, 024025 (2002) [arXiv:hep-th/0203039].

[14] E. Cremmer, S. Ferrara, L. Girardello and A. Van Proeyen, Nucl. Phys. B 212, 413 (1983).

[15] D. Z. Freedman, Phys. Rev. D 15, 1173 (1977); for a general formalism, see P. Binetruy, G. Dvali, R. Kallosh and A. Van Proeyen, Class. Quant. Grav. 21, 3137 (2004) [arXiv:hep-th/0402046].

[16] N. Arkani-Hamed and S. Dimopoulos, arXiv:hep-th/0405159; G. F. Giudice and A. Romanino, Nucl. Phys. B 699, 65 (2004) [arXiv:hep-ph/0406088]; A. Arvanitaki, C. Davis, P. W. Graham and J. G. Wacker, arXiv:hepph/0406034; A. Pierce, Phys. Rev. D 70, 075006 (2004) [arXiv:hep-ph/0406144]; C. Kokorelis, arXiv:hep-th/0406258; S. h. Zhu, Phys. Lett. B 604, 207 (2004) [arXiv:hep-ph/0407072]; B. Mukhopadhyaya and S. SenGupta, arXiv:hepth/0407225; W. Kilian, T. Plehn, P. Richardson and E. Schmidt, arXiv:hepph/0408088; R. Mahbubani, arXiv:hep-ph/0408096; M. Binger, arXiv:hepph/0408240; J. L. Hewett, B. Lillie, M. Masip and T. G. Rizzo, JHEP 0409, 070 (2004) [arXiv:hep-ph/0408248]; L. Anchordoqui, H. Goldberg and C. Nunez, arXiv:hep-ph/0408284; K. Cheung and W. Y. Keung, arXiv:hepph/0408335; N. Arkani-Hamed, S. Dimopoulos, G. F. Giudice and A. Romanino, arXiv:hep-ph/0409232; V. Barger, C. W. Chiang, J. Jiang and T. Li, arXiv:hep-ph/0410252; I. Antoniadis and S. Dimopoulos, arXiv:hep-th/0411032; A. Masiero, S. Profumo and P. Ullio, arXiv:hep-ph/0412058.

[17] S. Kachru, R. Kallosh, A. Linde and S. P. Trivedi, Phys. Rev. D 68, 046005 (2003) [arXiv:hep-th/0301240]; C. P. Burgess, R. Kallosh and F. Quevedo, JHEP 0310, 056 (2003) [arXiv:hep-th/0309187].

[18] R. Barbieri, S. Ferrara and C. A. Savoy, Phys. Lett. B 119, 343 (1982); A. H. Chamseddine, R. Arnowitt and P. Nath, Phys. Rev. Lett. 49, 970 (1982); L. J. Hall, J. Lykken and S. Weinberg, Phys. Rev. D 27, 2359 (1983); E. Cremmer, P. Fayet and L. Girardello, Phys. Lett. B 122, 41 (1983); N. Ohta, Prog. Theor. Phys. 70, 542 (1983).

[19] M. Dine, N. Seiberg and E. Witten, Nucl. Phys. B 289, 589 (1987); J. J. Atick, L. J. Dixon and A. Sen, Nucl. Phys. B 292, 109 (1987).

[20] A. Sagnotti, Phys. Lett. B 294, 196 (1992) [arXiv:hep-th/9210127]; E. Poppitz, Nucl. Phys. B 542, 31 (1999) [arXiv:hep-th/9810010]; L. E. Ibanez, R. Rabadan and A. M. Uranga, Nucl. Phys. B 542, 112 (1999) [arXiv:hep-th/9808139]; 
Z. Lalak, S. Lavignac and H. P. Nilles, Nucl. Phys. B 559, 48 (1999) [arXiv:hepth/9903160]; I. Antoniadis, C. Bachas and E. Dudas, Nucl. Phys. B 560, 93 (1999) [arXiv:hep-th/9906039]. 